\title{
OUTDOOR ADVENTURE THERAPY TO INCREASE PHYSICAL ACTIVITY IN YOUNG ADULT CANCER SURVIVORS
}

\author{
A Thesis \\ presented to \\ the Faculty of California Polytechnic State University, \\ San Luis Obispo
}

In Partial Fulfillment

of the Requirements for the Degree

Master of Science in Kinesiology

by

Elizabeth Catherine Gill

March 2015 
(C) 2015

Elizabeth Catherine Gill

ALL RIGHTS RESERVED 


\section{COMMITTEE MEMBERSHIP}

TITLE:

Outdoor Adventure Therapy to Increase Physical Activity in

Young Adult Cancer Survivors

AUTHOR:

Elizabeth Catherine Gill

DATE SUBMITTED:

March 2015

COMMITTE CHAIR: $\quad$ Suzanne Phelan, Ph.D

Associate Professor of Kinesiology

COMMITTEE MEMBER: Marni Goldenberg, Ph.D

Associate Professor of Recreation, Parks, and Tourism

Administration

COMMITTEE MEMBER: Heather Starnes, Ph.D

Assistant Professor of Kinesiology 


\section{ABSTRACT \\ Outdoor Adventure Therapy to Increase Physical Activity in Young Adult Cancer Survivors Elizabeth Catherine Gill}

Physical activity (PA) has numerous benefits for cancer survivors, but limited research exists on PA interventions in young adult cancer survivors. Outdoor adventure therapy is a potential method of increasing PA in this demographic. The primary purpose of this non-randomized parallel group study was to determine whether the outdoor adventure camp experience (vs. wait list control) would increase participants' PA levels immediately following the 7-day camp, as well as three months later. Secondary aims examined correlates of greater PA, including pre-post camp changes in sedentary behavior, exercise self-efficacy, environmental change self-efficacy, perceived barriers to exercise, physical activity enjoyment, and physical activity variety. Sixty-six control and 50 intervention participants were given validated quantitative questionnaires at baseline, 1 week (end of camp) and at the 3-month follow-up. Repeated measures multivariate analysis of variance (RMANOVA) was used to compare group changes over time. Using intent to treat analysis, adjusting for age, gender, age at diagnosis, and baseline minutes of PA, there was a significant difference ( $\mathrm{p}=.0001)$ in PA per week between groups at both 1 week and 3 months. Bonferroni adjusted post-hoc analysis indicated that, relative to baseline, the intervention group had significantly $(\mathrm{p}=.0001)$ greater increases in PA at both 1 week (577 minutes vs. 9 minute increases) and 3 month follow-ups (133 minute increases vs. 75 minute decreases; $p=.001$ ) respectively. Significant intervention-related improvements were also observed in TV viewing hours/week ( $\mathrm{p}=.001)$, hours sitting/week $(\mathrm{p}=.001)$, "Excuses" score of the Perceived Barriers to PA questionnaire $(\mathrm{p}=.04)$, Enjoyment of Structured Activities $(\mathrm{p}=.04)$, and PA Variety $(\mathrm{p}=.0001)$ at 1 week but not at the 3 month follow-up. No significant effects were observed for changes in exercise self-efficacy, environmental change self-efficacy, or the other subscales scores. In conclusion, outdoor adventure therapy has the potential to increase PA levels in cancer survivors both immediately following camp, as well as long-term. However, effects tend to wane after 
camp termination. Future research should explore the relationship between correlates of PA and PA levels in outdoor adventure therapy camp participants and methods to promote sustained PA after camp termination.

Keywords: young adult cancer survivors, physical activity, outdoor adventure therapy, correlates of physical activity 


\section{ACKNOWLEDGMENTS}

I would like to acknowledge my committee chair, Dr. Suzanne Phelan, for her invaluable feedback throughout this entire project. I am incredibly appreciative for her time and guidance throughout the study design, writing process, and data analysis. I have been lucky to learn from someone with her knowledge and research experience. I'd also like to thank my committee member Dr. Marni Goldenberg for her tireless support throughout the project. Without her time and energy from the beginning, this research project would not have been possible. Thank you also to committee member Dr. Heather Starnes for her time and support, and for the contribution of the Environmental Change Self-Efficacy Scale she developed.

I would like to acknowledge the financial support for this project through a CSU Agricultural Research Institute grant as well as a Shur Farms grant. I'd also like to express thanks to the organization First Descents for providing access to their participants for this study.

Last but certainly not least, I would like to express my heartfelt gratitude for all the young adult cancer survivors I've had the privilege of meeting who have inspired this thesis. 


\section{TABLE OF CONTENTS}

Page

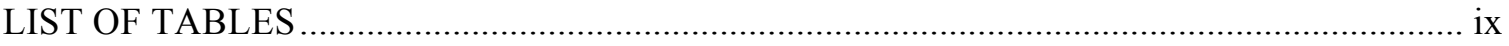

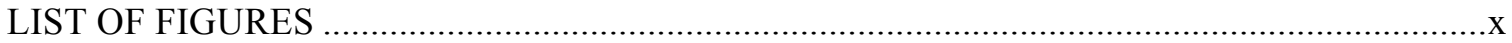

\section{CHAPTER}

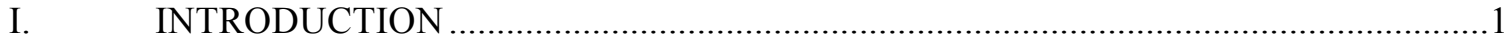

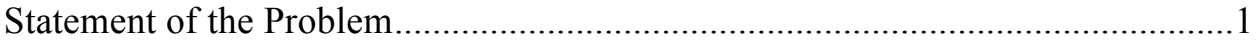

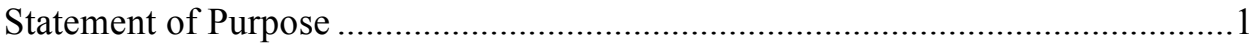

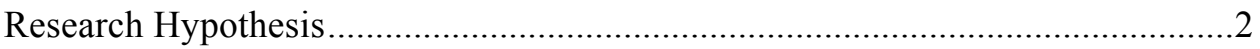

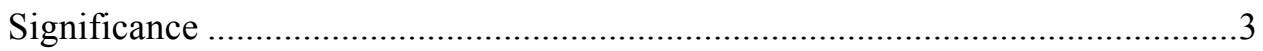

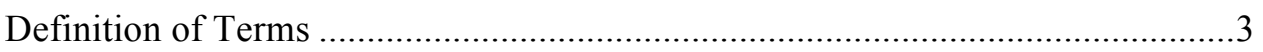

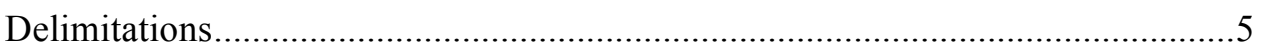

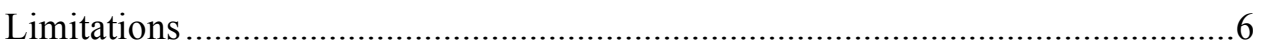

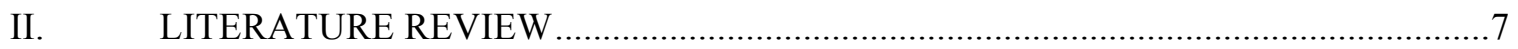

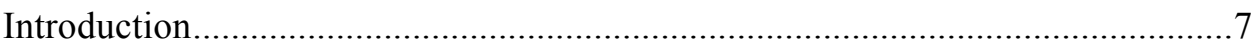

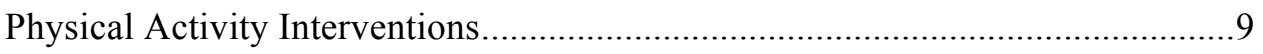

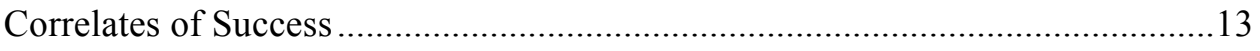

Summary and Rationale for Current Study ................................................. 17

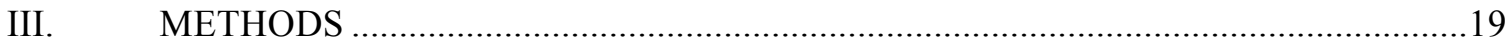

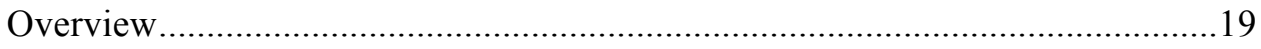

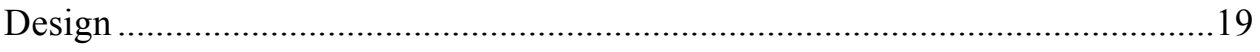

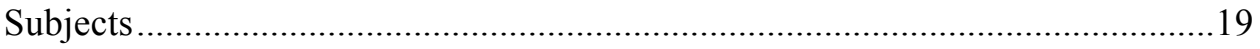

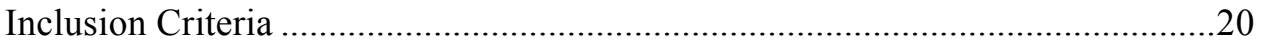

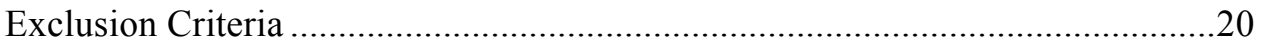

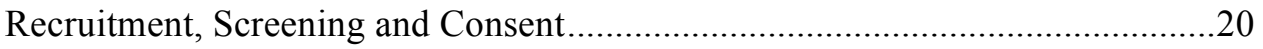

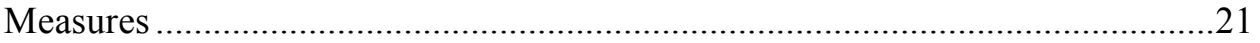




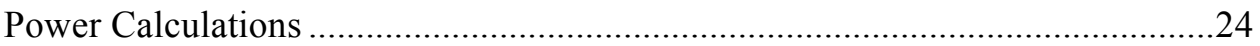

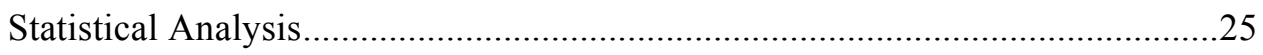

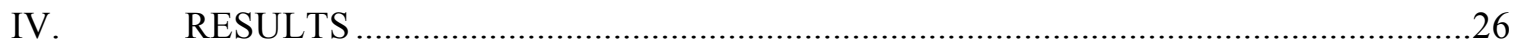

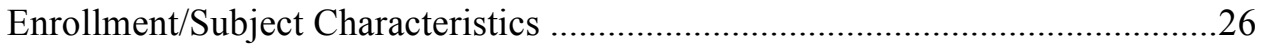

Retention At the 1 Week and 3 Month Assessment ............................................31

Minutes of Physical Activity per Week ............................................................... 31

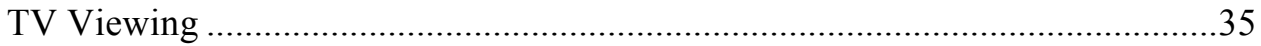

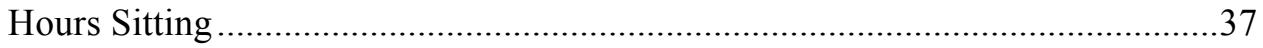

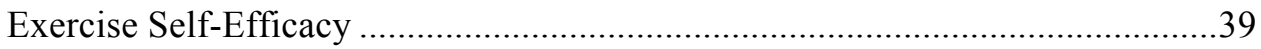

Environmental Change Self-Efficacy ........................................................4 41

Perceived Barriers to Physical Activity ............................................................4

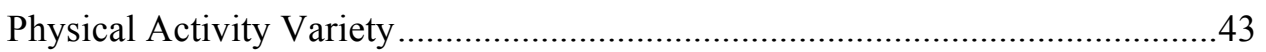

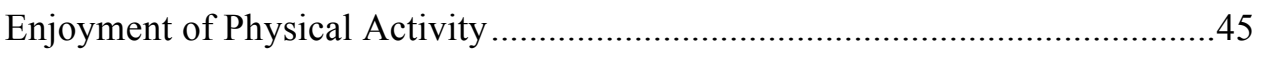

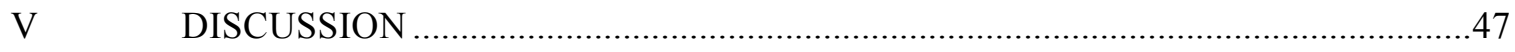

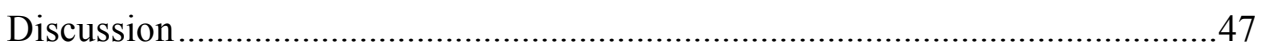

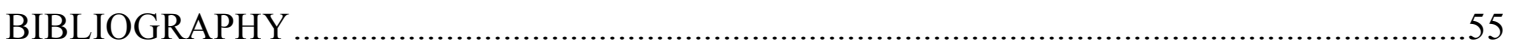

APPENDICES

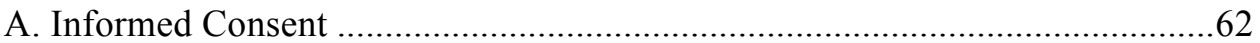




\section{LIST OF TABLES}

Table

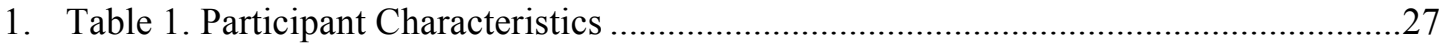

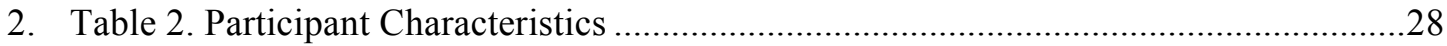

3. Table 3. Participant Cancer-Related Medical History ……................................................30

4. Table 4. Intent to Treat Comparison of Camp vs. Waitlist on Changes in Physical

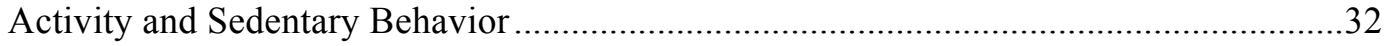

5. Table 5. Intent to Treat Comparison of Camp vs. Waitlist on Changes in Exercise Self-Efficacy and Environmental Change Self-Efficacy

6. Table 6. Intent to Treat Comparison of Camp vs. Waitlist Control on Changes in Perceived Barriers to Physical Activity

7. Table 7. Intent to Treat Comparison of Camp vs. Waitlist on Changes in PA Variety and Enjoyment of PA

8. Table 8. Intent to Treat Comparison of Camp vs. Waitlist on Changes in High or Low Preference for Vigorous, Moderate, or Inactive Recreation. 


\section{LIST OF FIGURES}

Figure

Page

1. Figure 1.1. Changes in Physical Activity over Time.....

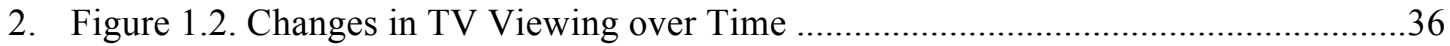

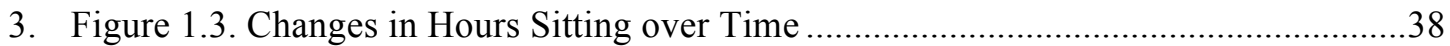




\section{Chapter 1}

\section{Introduction}

\section{Statement of the Problem}

Young adult cancer survivors (YACS) are an underserved demographic who have experienced little improvement in survival rates for the past 30 years (National Cancer Institute, 2012). Physical activity (PA) has the potential to improve cancer outcomes in this demographic, but surprisingly little research has been conducted in this area. Most of the existing research to date has been observational and primarily examined adult cancer survivors and children, but the effects of increases in activity in YACS are less documented (Rabin, Horowitz, and Marcus, 2013). Moreover, despite the overall health benefits of PA, average levels amongst cancer survivors remain low (Speed-Andrews, Stevinson, Belanger, Mirus, \& Courneya, 2012). Thus, there is a strong need to identify methods to increase exercise and examine effects in YACS (Speed-Andrews et al., 2012).

\section{Statement of Purpose}

The primary purpose of this non-randomized parallel group study was to determine if the First Descents camp experience (vs. wait list control) increased participants' PA levels during and immediately following the 7-day camp, as well as three months later. Secondary aims examined correlates of PA, including pre-post camp changes in self-efficacy, environmental change selfefficacy, perceived barriers to exercise, physical activity enjoyment, and physical activity variety. Researchers also explored changes in sedentary behavior, which have other important health implications (Katzmarzyk and Lee, 2012; Matthews et al., 2012) in addition to being a potential risk factor for certain types of cancer (Moore, Gierach, Schatzkin, \& Matthews, 2010).

The outdoor adventure therapy organization studied was First Descents, which is a nonprofit outdoor adventure therapy organization that provides free week-long experiences in outdoor adventure therapy, including surfing, whitewater kayaking, and rock climbing. Throughout the week-long program, participants are encouraged to push past their fear or 
reservations in order to embrace new outdoor adventure challenges throughout the week. Camp leaders are trained to foster the outdoor adventure therapy experience organically throughout the day's activities and in nightly recaps during campfires. Leaders encourage participants to embrace the concept of overcoming physical challenges and living life after cancer throughout the week. Camps take place in a group setting where participants are able to draw on support and experiences from their young adult cancer survivor peers. According to the First Descents (2014) website, YACS are empowered during the First Descents experience through "conquering legitimate outdoor challenges to push their limits and face their fears, and by doing so, they are able to regain the confidence and self-efficacy lost to cancer" (Who we Are section, para. 2). In addition, "the experience is designed to allow healing to happen naturally and organically - no forced conversations and no structured group sessions or therapy" (First Descents, 2014, Who we Are section, para. 2). Anecdotal evidence suggests that camp experiences result in meaningful and life-changing experiences for survivors by increasing their self-efficacy and encouraging them to live a more healthy and active lifestyle when they return home from camp (First Descents, 2014, Programs Testimonials section, Para. 2). However, formal evaluations of the camp have been minimal. The current study examined the impact on the camp experience on short and long-term (3-month) effects on physical activity, as well as correlates of PA.

\section{Research Hypothesis}

Specific Aims. Aim 1: Determine the short and long-term effects of an outdoor adventure therapy camp on PA levels/exercise.

Hypothesis: Relative to wait list controls, participants in camps would increase their PA levels during their experience and three months later. As a sub-aim, group differences in sedentary behaviors over the same time frame were examined.

Aim 2: To examine the effect of an outdoor adventure therapy camp on social cognitive constructs, including self-efficacy, environmental change self-efficacy, and perceived barriers. 
Hypothesis: Relative to wait list controls, participation in a camp would increase exercise selfefficacy and environmental change self-efficacy and would reduce perceptions of barriers to physical activity immediately following the camp and three months later.

Aim 3: To examine the effect of an outdoor adventure therapy camp experience on exercise variety and enjoyment of PA.

Hypothesis: Relative to wait list controls, participation in an outdoor adventure therapy camp would increase exercise variety and PA enjoyment immediately following the camp and three months later.

\section{Significance}

First Descents developed a method of programming aimed to increase PA levels of cancer survivors. However, little evaluative research has been done. Understanding the camps' effects on PA and related correlates is critical to strengthening First Descents' programming and highlighting possible ways the camp experience impacts health behavior. An increase in PA, even in a moderate amount, could benefit the physical health Moore et al. (2012) psychosocial health of cancer fighters and survivors. Findings from this study may advance understanding of ways to improve physical activity and psychosocial health in young adult cancer survivors.

\section{Definition of Terms}

Physical Activity (PA): PA was defined as any bodily movement produced by skeletal muscles that requires energy expenditure. For this study, PA also includes exercise, which is typically defined as a subcategory of PA defined as planned and repetitive and performed with the purpose of improving or maintaining components of fitness (World Health Organization, 2014). This could have included house chores, walking, gym activities, and sports. For this study it also included activities such as yoga, tai chi, and outdoor activities such as surfing, kayaking, and rock climbing. To count as PA, the effort of the activity must have been at a minimum be comparable to the effort of brisk walking. PA was recorded as either moderate, hard, or very hard. To count as moderate PA, the intensity must have been comparable to how it felt to walk briskly somewhere, 
while very hard intensity was similar to running intensity. The hard category was used to classify an activity falling in between moderate and very hard. PA was recorded by time of day (morning, afternoon, or evening) and intermittent or continuous activity was recorded per segment of the day. According to the Sallis Physical Activity Recall (Sarkin et al., 1997), if the activities add up to at least 10 minutes in one intensity category (moderate, hard, or very hard) for one segment of the day (morning, afternoon, or night), they were recorded. If they were spread out throughout the day, they were not recorded.

Environment: Environment referred to the physical or built environment where PA took place. This included the outdoor environment in open spaces, cities, and neighborhoods.

Cancer: Cancer referred to a term used for a disease in which abnormal cells divided without control and were able to invade other tissues (National Cancer Institute, 2012). Cancer included over 100 diseases broken down into broader categories including but not limited to: carcinoma, sarcoma, leukemia, lymphoma and myeloma, and central nervous system cancers. For the purpose of this study, cancer included all types of cancer.

Young Adult Cancer Survivors (YACS): YACS referred to people that survived or were currently fighting cancer and were in the 18-39 age range.

Outdoor Adventure Therapy: Outdoor adventure therapy referred to organized programs with planned outdoor activities that presented a physical and psychological challenge and presented an opportunity for self-growth. These programs typically took place in a group setting and activities could range from ropes courses to outdoor adventure sports such as surfing, whitewater kayaking, and rock climbing. The duration of programs could be anywhere from one day to several weeks or months depending on the intended purpose of the program and its participants.

First Descents: First Descents was a non-profit organization that provided YACS (18-39) free week-long outdoor adventure therapy experiences in surfing, whitewater kayaking, and rock climbing to help them overcome their cancer diagnosis. Programs focused on one outdoor adventure modality (surfing, whitewater kayaking, or rock climbing). They occurred in several 
locations throughout the US and worldwide. The organization primarily served first time participants, but has expanded to include camps for returning participants, participants aged 4049, and caregivers of YACS. This study examined first-time First Descents participants that attended a surfing camp in Santa Barbara, California, a whitewater kayaking camp in Jackson, Wyoming, or a rock climbing camp in Moab, Utah or Estes Park, Colorado.

Self-efficacy: Self-efficacy was defined as an individual's belief in his or her capacity to execute behaviors necessary to produce specific performance attainments Bandura (1977). This study specifically measured exercise self-efficacy, which was a measure of how confident one was in being able to exercise consistently. This study also measured environmental change self-efficacy, which referred to confidence in ability to seek out or create social and physical environments that supported PA.

Exercise Variety: Exercise variety referred to the different types of exercise (or PA) one engaged in throughout the week.

Exercise preferences: Exercise preferences referred to the category of intensity of exercise in which people preferred participating. The different categories were vigorous physical activities, moderate physical activities, or inactive recreational pastimes.

\section{Delimitations}

Participants in the study included YACS (18-39) that applied to attend a First Descents camp. These participants were limited to people who had heard about the organization through oncologists, young adult cancer service groups, word of mouth, or other methods. Participants in the program were also limited to those whom the organization selected for participation in a 2013 camp, while the waitlisted participants were those that applied, but were not selected for a 2013 program due to availability. Participants were residents of the U.S. and included those that were able to take a week of their time to attend the camp and participate in a week of kayaking, rock climbing or surfing. Ability to attend was based on the discretion of the organization, but participants at various stages of treatment and with varying medical needs were able to attend the 
camp. While the camp was free, some participants had to pay for their travel. Travel scholarships existed for those who could not afford to travel to the camp location.

\section{Limitations}

The non-randomized design of the study may have limited the extent to which observed changes can be definitively attributed to the camp. As participants in the study independently elected to apply to attend a First Descents camp, selection bias was also possible. Furthermore, participants' preference for camp location and modality was unmeasured. Participants might not have received their first choice due to camp size limitations, which may have influenced their outcomes of participation. Other limitations to the study included a lack of external validity to YACS participating in other exercise interventions or programs. There was a risk of loss of participants due to follow-up or attrition. Objective measures of activity were not used (e.g., autography). Data were collected by self-report, which has validity limitations in comparison to the current gold standard measure of an Actigraph Accelerometer. In a study comparing the Actigraph Accelerometer to the 7 Day PA Recall and IPAQ in breast cancer survivors, the validity correlation coefficient of total PA scores for the PAR was 0.73 and for the IPAQ was 0.33 Johnson-Kozlow, Sallis, Gilpin, Rock, and Pierce (2006). 


\section{Chapter 2}

\section{Literature Review}

\section{Introduction}

The medical and psychosocial burdens of cancer are well documented. Cancer survivors are at high risk of reduced life expectancy, cancer reoccurrence, and reduced quality of life (QOL) depending on stage and type of cancer as well as treatment regimens and a patient's overall health. The benefits of PA in ameliorating some of these burdens have been well documented in observational studies and include alleviating symptoms of treatment and improving QOL Solberg Nes et al. (2012). However, less is known about effective programs to increase activity, particularly in YACS. The primary purpose of this study was to examine whether outdoor adventure therapy can serve as an effective method of programming to increase PA levels and correlates of PA in YACS. This review will outline the benefits of PA for cancer survivors, current PA levels of cancer survivors, exercise interventions that have been tried, the limitations of tried interventions, and finally, how outdoor adventure therapy can serve as an effective exercise intervention in young adults.

Several population-based studies have documented the benefits of PA on improving the lifespan of adult cancer survivors. Data from a large prospective cohort of 632,091 US adults (median age of 61 years) found that increased leisure time PA significantly reduced the risk of premature death Moore et al. (2012). Similarly, in an observational cohort study of 2,897 women (aged 30 to 55 years) by Kroenke, Holmes, Feskanich, Chen, and Colditz (2005), findings indicated that higher levels of PA were related to improved chances of survival from breast cancer. Similarly, McTiernan et al. (2003) found that increased PA was related to reduced risk of developing breast cancer in 74,171 previously healthy women aged 50 to 79 . PA has the potential to prolong lifespan and prevent cancer incidence and reoccurrence in adult cancer survivors. 
Along with the health benefits, PA in survivors also appears to present psychosocial benefits, as documented in studies targeting older adults. According the American College of Sports Medicine (ACSM) Roundtable on Exercise Guidelines for Cancer Survivors, a review on the impacts of exercise on QOL found that four randomized controlled trials (RCT) of supervised exercise programs for breast cancer patients receiving chemotherapy and radiation therapy found that exercise improved QOL Schmitz et al. (2010). In breast cancer survivors that had completed chemotherapy or radiation treatment, 12 exercise interventions were found to improve QOL outcomes (Schmitz et al., 2010). A prospective observational study of US older adults (> 65) showed participating in regular PA of 150 minutes per week was associated with significantly improved QOL and alleviated symptoms than sedentary survivors (Solberg et al., 2012). Bélanger, Plotnikoff, Clark, and Courneya (2011) examined a random sample of 588 YACS (2044) and found that PA was strongly associated with QOL and found a significant dose-response association between PA and components of QOL such as depression, stress, and self-esteem. While PA is associated with psychosocial benefits in older cancer survivors, limited research has been conducted on the psychosocial benefits of PA on YACS.

Based on the documented benefits of PA for cancer survivors, the ACSM roundtable on exercise guidelines for cancer survivors concluded that PA was not only safe during and after cancer treatment, but that the recommended guidelines were equivalent with those for the general population. The recommended guidelines for cancer survivors is to engage in at least 150 minutes of moderate-intensity exercise, 75 minutes of vigorous-intensity exercise, or an equivalent combination weekly, as well as strength training and flexibility exercises two to three times a week, as recommended by the U.S. Departments of Health and Human Services Schmitz et al. (2010).

While no large-scale studies have evaluated the prevalence of PA in survivors, available evidence suggests that, despite these recommendations, current levels of PA in cancer survivors appear low. The American Cancer Society (ACS) states that cancer survivors are at increased risk 
for sedentary behavior because their PA levels decrease after diagnosis and during treatment, and rarely return to their pre-diagnosis levels (Doyle et al., 2006). In the Bélanger et al. (2011) study on the association between PA and QOL in YACS, only half of the survivors in the sample were meeting public health guidelines and a quarter of the participants were sedentary. A study comparing PA levels of 117 young adult survivors of childhood cancer vs. 148 age-matched healthy controls found that cancer survivors reported participating in moderate-to-vigorous PA (MVPA) 2.1 times fewer per week than controls Hocking et al. (2013). Thus, there is a strong need to identify methods to increase PA among cancer survivors (Speed-Andrews et al., 2012).

\section{Physical Activity Interventions}

Supervised exercise interventions. Exercise interventions that involved supervised exercise training sessions for cancer survivors in a gym or hospital setting have shown health benefits for cancer survivors, but most studies were in older adults. In a 12-week supervised exercise program, 26 colorectal cancer survivors with a mean age of 60 participated in two combined aerobic and resistance training sessions and one aerobic only session each week Sellar et al. (2014). Mean adherence to the exercise program was $91 \%$ and participants showed improvements in peak oxygen uptake, body strength, and a reduction in waist circumference. Broderick et al. (2014) studied de-conditioned cancer survivors with an average age of 51 that recently finished chemotherapy by randomly assigning them to a usual care group $(n=20)$ or an 8week aerobic based group exercise program $(n=23)$. The exercise program included supervised sessions twice a week in a hospital setting as well as an at-home exercise prescription. At the completion there was $78.3 \%$ adherence to the supervised exercise program and significant differences in physical well-being were found in favor of the exercise group at completion of the 8-week program and three months later. The exercise group had increased self-reported PA as compared to the usual care group at conclusion and three-month follow up. Improvements in fatigue, total QOL, and physical functioning were found at the three-month follow up in the exercise group. Supervised exercise interventions in cancer survivors have found positive results 
in exercise adherence and physical functioning, but research examining effects of supervised exercise programs in young adults is lacking.

Home based interventions. Home-based PA interventions also had positive outcomes for cancer survivors. A 12-week home-based exercise intervention randomized 43 breast cancer survivors with an average age of 53 to either a control or an exercise group that consisted of a moderate-intensity exercise program (participants were asked to target a certain percent of estimated maximum heart rate) with a goal of increasing duration each week (Pinto, Rabin, \& Dunsiger, 2009). In addition, participants in the exercise group were given telephone counseling each week based on Transtheoretical Model (TTM) and Social-Cognitive Theory (SCT) as well as two exercise tips per week in the mail. While no exercise adherence data was collected on the control group, breast cancer survivors in the exercise group significantly increased minutes of exercise and steps taken from week one to the conclusion of week 12. Basen-Enquist et al. (2013) enrolled 100 women in a 6-month home-based exercise treatment program where participants were given moderate-intensity exercise goals consistent with ACSM guidelines as well as telephone counseling and print materials supporting PA. Findings indicated positive effects on average minutes of PA per day at the two, four, and 6-month assessment period as compared to baseline minutes of PA.

Other home-based interventions have found mixed results at long-term follow-ups to PA interventions. Forty-six colorectal cancer survivors with a mean age of 57 were randomized to either a 12-week control group or a home-based PA intervention that provided TTM and SCT based counseling by phone and mail (Pinto, Papandonatos, Goldstein, Marcus, \& Farrell, 2013). This study found that the PA group had significant increases in PA at three months as compared to a control group, but the differences between groups were attenuated at 6 and 12 months postbaseline. In addition, there were no significant group effects on psychosocial outcomes at the three, 6, or 12-month assessments. Rogers et al. (2009) measured maintenance outcomes three months post-completion of a 12-week physical activity intervention in which 41 breast cancer 
survivors (average age 53) received supervised group and individual sessions before being tapered to a home-based exercise program after 6 weeks. The intervention aimed at targeting SCT variables. Findings indicated that at three months post-intervention, participants showed an improvements in PA levels, muscular strength, central adiposity, and social well being. Future research examining home-based interventions could benefit from more in depth examination of outcomes at follow-up and more diverse samples of participants, including YACS.

Alternative exercise interventions. Alternative exercise interventions showed promise for increasing PA in cancer survivors, but research designs were exploratory in nature. Twenty three post-adjuvant therapy breast cancer survivors participating in a community 12 -week Iyengar yoga program twice a week were found to have $63.9 \%$ adherence to the program (Speed-Andrews et al., 2012). Despite the short-term nature of the study, homogenous sample, and the fact that participants were already enrolled in the yoga program, the findings showed promise for Iyengar yoga as an effective way to increase PA adherence in cancer survivors. Tai chi was also a possible alternative form of exercise that could increase QOL and overall PA in cancer survivors. Twenty-one breast cancer survivors randomized to either a 12-week tai chi chuan exercise group or a standard support group (both exercising three times per week for 60 minutes a session) were tested for effects of tai chi on overall exercise and health related quality of life (HRQOL) and biomarkers associated with side effects of cancer and cancer treatment (Sprod et al., 2012). Adherence to the tai chi exercise intervention was $72 \%$ vs. $67 \%$ in the standard support group. Results indicated that tai chi chuan significantly improved HRQOL, physical functioning, physical role limitations, social functioning, and general mental health.

Carter et al. (2012) hypothesized that team-based PA would be more effective than group-based PA by having cancer survivors choose between two similarly structured 8-week exercise program: a team-based dragon boat team $(n=68$, average age 53.8) or a group-based walking program ( $\mathrm{n}=52$, average age 52$)$. Findings indicated that the team-based group, as hypothesized, had significantly greater short-term outcomes of team cohesion and program 
adherence based on the group-based nature of the dragon boating activity. Participants in dragon boating also had improved upper body strength as compared to the walking program. Both groups showed improvements in physical fitness and QOL outcomes as compared to baseline, but there were no significant differences between the two groups.

Only one study to date has tested the effects of an intervention to increase PA in YACS. Valle, Tate, Mayer, Allicock, and Cai (2013) examined efficacy of a social media Facebook exercise intervention. Eighty-six YACS were randomized to either a 12-week Facebook-based intervention FITNET (average age 30.8) or a Facebook-based self-help comparison condition (average age 32.7), and participants were assessed on physical activity. The FITNET intervention group received a PA goal, tips related to PA, and additional intervention components based on SCT framework. Both groups reported an increase in self-reported minutes of MVPA per week and there was no significant difference in MVPA between the 2 groups. However, the FITNET group reported significant increase in weight loss over time and increases in light physical activity of $135 \mathrm{~min} /$ week greater than the SC group. More research is needed to test the efficacy of such interventions at increasing exercise adherence and PA correlates.

Theory-based interventions. Most exercise intervention studies in cancer survivors have lacked a theoretical basis, but there are notable exceptions. Pinto et al. (2009) used both Transtheoretical model and Social Cognitive Theory and, consistent with hypothesis, found that an increase in self-efficacy was significantly related to increase in PA. Rogers et al. (2009) utilized SCT in their intervention and hypothesized that the positive outcomes on PA levels, physical fitness and social well-being were due to a theory based-intervention designed based on careful attention to the needs and preferences of the target population. While Broderick et al. (2014) did not use a theory-based intervention, improvements in QOL and fatigue were linked with improvement in aerobic fitness and PA levels. Mutrie et al. (2007) did not report a specific theoretical framework, but the authors also reported that functional and psychological benefits such as breast cancer specific quality of life and positive mood were found after a 12-week 
supervised exercise program. Theories that propose methods to increase PA can potentially be utilized by interventions looking to increase PA in cancer survivors.

In cancer survivors, the most common models used in theory-based interventions include SCT, the Theory of Planned Behavior (TPB), and the TTM. Interventions utilizing SCT/SelfEfficacy Theory found that a higher level of self-efficacy was strongly correlated with higher levels of exercise behavior immediately post-intervention and long-term (Basen-Engquist et al., 2013; Rogers et al., 2009). Further research in the cancer population is needed to determine if self-efficacy is a determinant of PA (Basen-Engquist et al., 2013). Interventions modeled on the TPB framework demonstrate that intention is the strongest TPB variable linked to exercise behavior (Speed-Andrews et al., 2012). In addition, TPB may be a useful framework for future studies examining novel ways to increase PA in cancer survivors, such as yoga programs (SpeedAndrews et al., 2012). TTM-based interventions have been successful in increasing exercise behavior in this population short-term, but existing research shows mixed results on whether these changes are maintained long-term (Loprinzi, Cardinal, Si, Bennett, \& Winters-Stone, 2012; Pinto et al., 2009).

Limited research exists on exercise interventions in cancer survivors that utilize theoretical frameworks and health behavior change models. More research is needed in more diverse samples of cancer survivors that include different cancer types and participant ethnicities, different types of exercise programs, and studies that measure short-term and long-term maintenance of outcomes.

\section{Correlates of Success}

Successful adoption and maintenance of a supervised exercise intervention can be more likely when the intervention addresses and increases correlates of PA such as self-efficacy in participants (Loprinzi et al., 2012). Self-efficacy is considered a major determinant of PA and exercise adherence. Self-efficacy is defined as the beliefs an individual has about ability to engage in behaviors that lead to expected outcomes (Bandura, 1977). According to SCT, self- 
efficacy improves health habits and behavior change directly and indirectly through improving outcome expectations, goals and sociostructural factors (Bandura, 2004), including reducing barriers and increasing facilitators. Exercise self-efficacy is defined as one's confidence in being able to make time for exercise and maintain regular exercise in the face of a variety of obstacles. Loprinzi et al. (2012) conducted a prospective RCT of 69 breast cancer survivors (with a mean age of 71) divided into an aerobic exercise, resistance exercise, or a control group of stretching and relaxation exercises. Participants exercised for 60 minutes at a time, three times a week, for one year. At the conclusion of the program participants were given a 6-month training program complete with equipment and an instructional DVD. Loprinzi et al. found that participants with higher self-efficacy and higher behavioral processes of change at the conclusion of the supervised exercise program reported higher levels of PA at the 6-month follow up. In the Pinto et al. (2009) intervention breast cancer survivors in the intervention group were given tips based on TTM and SCT, and exercise self-efficacy was found to significantly predict exercise adherence. In a longitudinal study of endometrial cancer survivors, Basen-Engquist et al. (2013) found selfefficacy to be a strong predictor of minutes of PA. Having higher morning self-efficacy significantly predicted the participant's total exercise minutes for the day and exercise selfefficacy was the only SCT variable that predicted exercise at the next measurement. Exercise adherence was strongly predicted by TPB variables such as stronger intention, greater selfefficacy, and more positive instrumental attitude (Speed-Andrews et al., 2012).

Other correlates of PA such as PA preferences, enjoyment of PA, barriers to PA and sedentary behavior have been associated with successful exercise interventions in cancer survivors. Basen-Engquist et al. (2013) measured SCT variables and while they found exercise self-efficacy to be associated with PA, barriers self-efficacy was not significantly associated with PA. In cross-sectional study of 192 breast cancer survivors measuring correlates of PA, barriers self-efficacy was found to have significant and direct associations with perceived PA barriers, enjoyment, social support, and current leisure PA (Rogers, McAuley, Courneya, \& Verhulst, 
2008). In a study measuring PA preferences of 175 non-small cell lung cancer survivors, $83 \%$ of survivors reported preferring low to moderate PA, but more research is needed in preferred exercise intensities of different types of cancer survivors and YACS (Philip et al., 2014).

Reducing sedentary behavior has the potential to improve health outcomes in cancer survivors. While no large-scale studies have examined the long-term effects of sedentary behavior in cancer survivors, sedentary behavior has been proven to have harmful health risks for the general population. A prospective study of 17,013 healthy Canadians found that there was a dose-response association between sitting time and mortality from all causes and cardiovascular disease independent of leisure-time PA (Katzmarzyk, Church, Craig and Bouchard, 2009). Reducing sedentary behavior in cancer survivors has the potential to decrease the occurrence of health risks associated with sedentary behavior in the general population.

More research is needed to explore the relationship between sedentary behavior and PA in cancer survivors. The research between sedentary behavior and PA is complex and poorly understood in healthy individuals and cancer survivors. In the general population, an observational study found that sedentary behavior in the form of computer use was associated with a higher level of physical inactivity in 18-30 year olds and that a significant proportion of those with moderate to high levels of computer use reported that this acted as a barrier to PA (Fotheringham, Wonnacott, \& Owen, 2000). However, other studies have found no relationship between activity and sedentary time. An observational study of 2,650 adults found that while leisure-time computer use is strongly related to being overweight, it is largely independent of leisure-time PA (Vandelanotte, Sugiyama, Gardiner, \& Owen, 2009).

In cancer survivors, Wijndaele et al. (2009) found that higher levels of television viewing time were associated with higher BMIs two and three years post-diagnosis in a prospective study of 1,867 colorectal cancer survivors. Findings suggest that reducing TV viewing could lead to less weight gain after diagnosis, which could subsequently reduce risk of comorbid conditions such as Type 2 diabetes and cardiovascular disease. In a study of 111 female breast cancer 
survivors that wore an accelerometer for 7 days, findings showed that light intensity PA was negatively associated with adiposity, while sedentary behavior was positively associated with adiposity, suggesting that increasing light and moderate PA might displace sedentary time and improve other health outcomes for cancer survivors (Lynch et al., 2010).

Surprisingly, limited studies exist on the correlates of environmental change self-efficacy and PA variety in cancer survivors, although they have been found to be associated with PA in other populations (Bond et al., 2012; Ryan \& Dzewaltowski, 2002). Environmental change selfefficacy is an innovative and emerging new concept that posits that people's beliefs in their ability to find and create social and physical environments is critical to improving PA rates. Although research in this area is in its infancy, a study comparing the relationships between different types of self-efficacy and PA in youth found that environmental-change efficacy was the strongest correlate of youth PA (Ryan \& Dzewaltowski, 2002). Another factor linked with more activity is PA variety, or the number of different types of PA in which people are engaged. PA variety has been shown to be positively related to greater amounts of objectively measured PA in adults without a history of cancer (Bond et al., 2012).

While limited research exists testing the effects of PA interventions in YACS, several qualitative studies have explored treatment preferences of this demographic. Rabin et al. (2013) conducted semi-structured interviews in an exploratory qualitative study with 20 YACS about intervention preferences and the emerging themes were that interventions should work with competing obligations (such as work and family) and that interventions should also provide social support. Zebrack, Bleyer, Albritton, Medearis and Tang (2006) studied 37 YACS to assess health and supportive care needs and found that $96 \%$ ranked meeting other survivors as a top five need. In a later study, Zebrack (2009) assessed 879 18-39 year olds diagnosed with cancer using surveys and found that upwards of $60 \%$ of the participants expressed a desire for programs offering services such as age-appropriate cancer information, complementary and alternative health services, and camp or retreat programs for young adults. Rabin et al. (2013) extended the 
work of Zebrack's study to conduct an in-depth qualitative study with 20 YACS and found that programs involving PA, relaxation, emotional support, information, nutrition/weight management, and similarity with other participants were the common program categories that might promote physical and emotional health for YACS. Taken together, there appears to be a strong need for interventions tailored to the needs of this demographic.

\section{Summary and Rationale for Current Study}

Most of the exercise interventions to date have examined supervised exercise programs for older adults in a gym or hospital setting, or home-based exercise activities through walking or exercise DVDs. These interventions have been studied in older cancer survivors typically 50 years and older. However, such programs might not appeal to a younger demographic who, in qualitative research, have reported a desire for more social activity PA programs such as outdoor activities like surfing, kayaking, rock climbing, or trail running/hiking. In addition, a theorybased intervention could prove successful in this demographic. The YACS demographic has been described as a unique demographic with different physical and psychosocial needs than children or adults due to the transitional nature of adulthood, such as different employment, social, financial, medical, and residential stability (Zebrack, 2009). While the YACS group may has been a challenging demographic to research due to frequent change of address or lack of medical insurance, as reported in Rabin et al. (2013), innovative methods are needed to increase PA and improve the health outcomes of this demographic.

Outdoor adventure therapy has the potential to serve as a method of increasing PA in YACS. This method engages participants in an outdoor activity including hiking, rock climbing, and other outdoor sports. The process of mastering and overcoming an unfamiliar physical challenge in an unknown environment has been hypothesized to improve self-concept through Mental health promotion (MHP), a strategy to promote health and provide strategies to recover from adversity (Epstein, 2004). Rosenberg, Lange, Zebrack, Moulton and Kosslyn (2014) found that 87 YACS participating in a one-week outdoor adventure therapy camp improved their body 
image, self-compassion and self-esteem and reduced their depression and alienation relative to pre-test and compared with a control group. These results were significant after attending their first camp, but not their second time attending camp. Ten days of adventure therapy resulted in positive improvements in the emotional, physical, and psychological rehabilitation of 11 adolescents with cancer and found emerging themes of developing connections, togetherness, rebuilding self-esteem, and creating memories Stevens et al. (2004). Sugerman (2005) conducted a qualitative study on 4 female cancer survivors aged 45-57 participating in a one-day outdoor challenge course program and found that the three emerging themes from the data were that participants emerged with renewed sense of self, feeling of support, and sense of control. Adventure therapy may be particularly beneficial for YACS who might be especially open to learning about and adopting new behaviors if taken out of their day-to-day environment and away from their commitments and stressors from work, family, and social obligations. However, controlled studies of the effects of outdoor adventure therapy in YACS have not been conducted. 


\section{Chapter 3}

\section{Methods}

\section{Overview}

This study examined the effects of an outdoor adventure therapy program on PA levels and PA correlates of young adult cancer survivors. This is a co-study of a qualitative study by Dr. Marni Goldenberg of the Cal Poly San Luis Obispo Recreation, Parks, and Tourism Administration Department that is measuring outcomes of participation in the outdoor adventure therapy program. In this quantitative co-study, a target sample size of 60 outdoor adventure therapy camp participants were recruited and compared with wait list control and followed for five months and assessed at one month pre-camp, immediately post-camp (end of camp), and three months post-camp.

\section{Design}

An observational prospective two group parallel design was used. Participants from two rock climbing, two surfing, and two whitewater kayaking camps were compared with a control group of cancer survivors who applied to attend a 2013 outdoor adventure therapy program but were waitlisted. Assessments were done one month pre-camp, immediately post-camp, and three months post-camp.

\section{Subjects}

A target sample size of 60 camp participants was selected with plans to recruit an equal number of participants from each camp modality: rock climbing, surfing, and whitewater kayaking. Recruitment was targeted at two rock climbing camps in Moab, Utah, and Estes Park, Colorado $(\mathrm{N}=20)$, two surfing camps in Santa Barbara, $\mathrm{CA}(\mathrm{N}=20)$, and two whitewater kayaking camps in Jackson, Wyoming $(\mathrm{N}=20)$. A target sample size of 60 control participants was selected with plans to recruit from the First Descents waitlist from the national population of camp applicants. Subjects were recruited from the outdoor adventure therapy program First Descents, a 
non-profit organization that serves YACS (18-39) by providing free week-long outdoor adventure therapy experiences in whitewater kayaking, rock climbing, and surfing.

\section{Inclusion Criteria}

The inclusion criteria for the study were that all participants were 18-39 year old cancer survivors or fighters and included males and females with a prior diagnosis of cancer of any type. The participants consisted of applicants to First Descents. First Descents only included young adults with a previous diagnosis of cancer of any type. Participants were accepted whether they were in remission or currently receiving treatment for cancer. Participants of varying medical conditions, including mobility impairments, amputees, vision impairments, seizure disorders, or with special treatment or diet needs were accepted into the program. National applicants to the organization were placed on their waitlist if they met the program criteria, but there was a lack of space in programs for the 2013 season. They were selected for a program if there was a lastminute cancellation or would potentially be selected for the 2014 season.

\section{Exclusion Criteria}

Participants were not accepted into the organization First Descents if they were outside the age range of young adults (18-39), attended a First Descents camp before, or had medical conditions that would prevent them from being able to safely travel to and attend a camp and participate in the outdoor activities of whitewater kayaking, surfing, or rock climbing. This study had no exclusion criteria beyond those of First Descents.

\section{Recruitment, Screening, and Consent}

Camp participants were enrolled in an ongoing qualitative study that examined immediate outcomes of the First Descents experience using means-end theory, a qualitative research technique known as laddering (Reynolds \& Gutman, 1988). The qualitative study also was also designed to examine the impact of the outdoor adventure camp on broadening environmental awareness. Consent to participate in the proposed co-study was integrated into the consent form for the qualitative study. 
As for the current study, First Descents agreed to screen applicants for eligibility in participating in a First Descents program as well as select participants that have never attended a First Descents camp. From this population, the organization gave researchers access to potential participants from six camps from late in the 2013 program season (August until September, 2013) as well as the wait list. These selected participants were approached by the organization First Descents in an email eight weeks before the start of their selected camp to inform them about the study. The email provided an introductory letter from the researchers and an electronic consent form. The organization sent a reminder email one week later to the participants in the study with the same introductory letter and link to the electronic consent form. Participants that selected to participate in the study by signing the consent form were enrolled in the study and contacted by the investigator of this study. The investigator called the participants to describe the study in detail and administer the pre-camp assessments: 7-Day PA Recall via phone and the Demographics and Questionnaire Packet electronically. The investigator met with participants from the First Descents camp at the end of the week-long program and only spoke with control participants by phone and electronically. The Human Subjects Institution Review Board at Cal Poly approved this study.

\section{Measures}

For this study, assessments were conducted electronically (and over the phone for PA recall) at three time points: one month prior to camp, immediately following the 7-day camp, and three months after the end of the camp.

Participant demographics and medical history. A self-administered survey measured age, height, weight, race/ethnicity, marital status, education, smoking history, alcohol use, cancer diagnosis, stage of cancer, age at diagnosis, time since diagnosis, time since treatment, cancer treatment (radiation, chemotherapy, hormonal therapy, surgery), current medications, comorbid conditions, remission status, and various other questions related to their treatment history. The participant demographics form was adapted from the First Descents physical exam and 
participant application forms. These forms contained demographic questions that were commonly used in research covering YACS and PA interventions in older cancer survivors Ottenbacher et al. (2011); (Zebrack, 2009). A list of comorbid conditions was adapted from the comorbid conditions demographics questionnaire used in the breast cancer study on SCT and PA by Rogers et al. (2005) and adapted from the study by Groll, To, Bombardier and Wright (2005).

Participants were given the full demographics survey pre-camp, and were asked if any of this information had changed at the two post-camp assessments.

Physical activity levels. The scale used to measure PA was the 7-Day PA Recall (PAR), a validated quantitative questionnaire that was conducted over the telephone by a trained interviewer in order to collect data on PA (including minutes of different PA intensities and total kilocalories of energy expenditure), and hours of sleep over the course of the past seven days (Sallis et al., 1985). The 7-Day PAR was originally developed for use in the Stanford Five-City Project in 1985 and has been widely used in epidemiologic, clinical, and behavior change studies (Sarkin et al., 1997).

The 7-Day PAR was used effectively in studies measuring PA in cancer survivors (Pinto et al., 2013; Ottenbacher et al., 2011). The 7-Day PAR was proven more effective than the International Physical Activity Questionnaire (IPAQ) in breast cancer survivors in terms of validity correlation, sensitivity, specificity, and was found to be superior to the IPAQ in terms of validity, measurement bias, and screening statistics (Johnson-Kozlow et al., 2006).

Sedentary behavior. The sedentary behavior questionnaire consisted of two questions pertaining to number of hours spent watching television and number of hours spent seated (not including TV watching) in the previous seven days (Salmon, Owen, Crawford, Bauman, \& Sallis, 2003).

Exercise self-efficacy. To measure self-efficacy amongst participants, an exercise selfefficacy scale was used to measure perceived efficacy related to the specific task of exercise and physical activity. According to Bandura's Social Cognitive Theory, self-efficacy was the most 
powerful determinant of behavioral change because self-efficacy expectancies determine the decision to perform a behavior and continue performing that behavior against adversity (Sherer et al., 1982). The James Sallis Self-Efficacy and Exercise Habits Survey was developed as a 12-item scale that asks participants to rate their confidence in motivating themselves to overcome barriers to exercising for at least six months (Sallis, Grossman, Pinski, Patterson, \& Nader, 1987). The questionnaire was rated on a 5-point scale with the anchors "I know I cannot" and "I know I can" and included items such as asking about confidence in maintaining an exercise program "when undergoing a stressful life change (e.g. divorce, death in the family, moving)." The scale produced two scores: "Making time for exercise" and "Sticking to it." This scale was developed to study the mediating effects of self-efficacy in exercise behavior change studies.

Environmental-change self-efficacy. An environmental-change self-efficacy questionnaire developed by Dr. Heather Starnes of the Cal Poly, San Luis Obispo, Kinesiology Department was used to determine participants' confidence in being able to participate in physical activity in their current environment. This scale included questions such as, "How confident are you that: you can find a trail/path near your home where you can be physically active, you can ask your community leaders to create places (e.g., trails, parks, bike lanes, playgrounds, sports fields, fitness centers, etc.) to be physically active near your home, and you can ask your family members/roommates to make room for physical activity in the common areas of your home?" The items were scored on a 0-4 likert scale and the scale produced one score. This scale was a newly developed measure with no validity or reliability data published to date.

Perceived barriers to exercise. A perceived barriers to exercise questionnaire was used to assess perceived barriers to exercise. The James Sallis Barriers to PA Scale from the Project Grad Health Assessment Survey was a validated quantitative questionnaire (Calfas et al., 2000). The question "How often do the following prevent you from getting PA?" was followed by a list of items that included lack of time, lack of energy, family demands, etc. The scale produced an overall perceived barriers to exercise score as well as a score for Aversiveness, Inconvenience, 
Worries, Excuses and Demands. Cancer-specific questions from Lynch (2010) on barriers to PA included items such as "My doctors do not encourage me to do more physical activity" and "Diarrhea or incontinence makes it difficult for me to be more physically active." These items were scored on a 5-point likert scale. This scale produced a cancer-specific score.

Physical activity enjoyment. PA enjoyment measures consisted of the questionnaires Preferred Activities, Enjoyment of PA, and Enjoyment of Inactive Recreation. All three questionnaires were validated quantitative questionnaires (Salmon et al., 2003). The Preferred Activities Questionnaire asked participants whether they preferred to do moderate PA, vigorous PA, or inactive recreational activities at different points of the day. The Enjoyment of PA Questionnaire asked participants to rate their level of enjoyment 12 physical activities that included cycling and digging in the garden on a 5-point likert scale. The Enjoyment of Inactive Recreation scale asked participants to rate their enjoyment of 9 different inactive recreational activities such as talking on the telephone or using a computer on a 5-point likert scale.

Physical activity variety. During the 7-Day PA Recall assessment, the researcher also captured PA variety by recording a list of the past week's physical activities for each participant.

\section{Power Calculations}

To conduct a power analysis to determine sample size, calculations were based on a study by Valle et al. (2013) that examined a similar sample (86 YACS) in which PA was measured via self-report by a validated quantitative questionnaire (the Godin Leisure Time Exercise Questionnaire). In Valle et al. (2013), 12-week significant changes in minutes/week of light PA between intervention and control were found with an unadjusted mean change of $163.6 \pm 350$ in the treatment group and $28.5 \pm 92.5$ in the control group (average SDs used). Assuming the same magnitude of difference and 120 participants (60 in each group), this study would have $82.4 \%$ power to detect a significant effect on PA between two groups, with a twotailed test and alpha $<0.05$. 


\section{Statistical Analysis}

Data were entered into excel and exported into SPSS statistical software for analysis. A baseline comparison of two groups was completed with chi squared analysis. Intent to treat analysis was used with all available data, assuming baseline values for missing data.. Sample sizes in some of the models differed due to missing baseline data on some questions. For missing baseline data on age $(n=6)$ and age at diagnosis $(n=11)$, group averages were assumed. Repeated measures multivariate analysis of variance (RMANOVA) was used to compare First Descent participants and control participants on changes in PA levels, exercise self-efficacy, environmental change self-efficacy, perceived barriers to exercise, PA enjoyment, PA variety, and sedentary behavior across time. Follow-up independent $\mathrm{t}$ tests using Bonferroni adjusted post-hoc analysis were used to compare significant changes from baseline to 1 week and baseline to 3 month follow-up. All analyses were adjusted for baseline values, age, gender, and age at diagnosis. Analyses adjusted for cancer stage revealed similar findings, but were not included in model due to amount of participants missing a cancer stage $(n=25)$. Logistic regression analysis was used to examine associations with preference for PA (high vs. low) post-camp and at the 3 month follow-up, adjusting for the same covariates. Partial correlation analysis was used to explore the relationship between changes in PA and sedentary behavior. 


\section{Chapter 4}

\section{Results}

\section{Enrollment/Subject Characteristics}

Of the potential participants approached by the organization about the study, 69\% (149 of 216) were eligible and consented to participate. Enrollment of 149 exceeded expected sample size of 120 . Of those who consented, $78 \%$ (116 of 149) completed the baseline questionnaire. More participants in First Descents (50/51) than in Control (66/98) completed the baseline questionnaire. The only difference in baseline characteristics between the two groups was age at diagnosis with the control group having a slightly higher age at diagnosis $(30.13 \pm 7.56$ vs. $27.34 \pm 6.02 ; \mathrm{p}=.040)$. There were no significant differences in other characteristics.

As shown in Tables 1 and 2, overall, subjects had an average age of $32.42 \pm 6.52$ years old, had a BMI of $24.95 \pm 4.43 \mathrm{~kg} / \mathrm{m} 2$, and were $79.3 \%$ female and $88.1 \%$ Caucasian. Subjects had an average of $3.35 \pm 3.78$ years since diagnosis of cancer. Overall, participants reported few total activity restrictions (including lymphedema, neuropathy, musculoskeletal weakness, limited range of motion, or joint pain) and comorbid conditions (including arthritis, osteoporosis, chronic obstructive pulmonary disease, acquired respiratory distress syndrome, emphysema, asthma, angina, congestive heart failure or heart disease, heart attack, neurological disease, stroke, peripheral vascular disease, diabetes type I or II, upper gastrointestinal disease, depression, anxiety or panic disorders, visual impairment, hearing impairment, degenerative disc disease, obesity, eating disorder). As shown in Table 2, most subjects were working (68.5\%), a minority were attending school (19.4\%), and less than half were married $(43.2 \%)$ or with children $(37.6 \%)$. The majority of participants received a bachelor's degree $(38.3 \%)$ or graduate degree $(30.8 \%)$. 


\begin{tabular}{|l|l|l|l|}
\hline \multicolumn{2}{|l|}{ Table 1 } \\
\multicolumn{2}{|l|}{ Participant Characteristics } & \multicolumn{1}{l|}{ Intervention } & $p$ \\
\hline Characteristic & Control & $31.12 \pm 5.498(n=50)$ & .06 \\
\hline Age (years) & $33.50 \pm 7.120(n=60)$ & $25.84 \pm 5.06(n=50)$ & .05 \\
\hline BMI (kg/m2) & $24.17 \pm 3.67(n=57)$ & $27.34 \pm 6.02(n=50)$ & .04 \\
\hline Age at diagnosis & $30.13 \pm 7.56(n=55)$ & & \\
\hline (years) & & $3.49 \pm 3.52(n=50)$ & .73 \\
\hline Time since diagnosis & $3.24 \pm 4.02(n=58)$ & & \\
\hline Total comorbid & & & \\
\hline conditions & $1.21 \pm 1.47(n=66)$ & $1.14 \pm 1.11(n=50)$ & .76 \\
\hline Total activity & & & \\
\hline
\end{tabular}




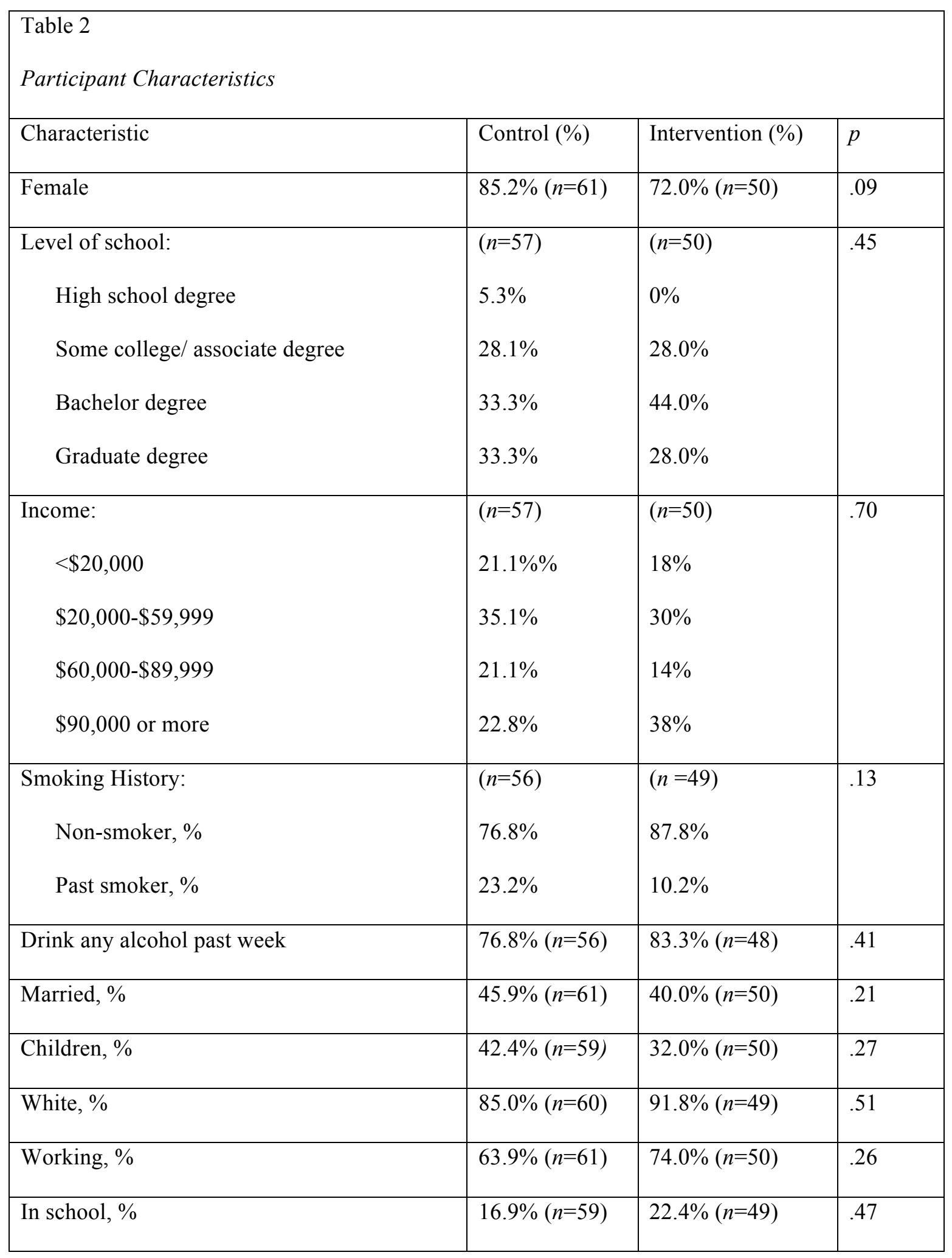


As shown in Table 3, most participants (80.6\%) reported currently taking medications. Most participants also reported a past history of treatment in the form of chemotherapy $(82.2 \%)$, radiation therapy $(56.0 \%)$, or a history of surgeries $(87.7 \%)$. The majority of subjects were in remission (61.0\%), and a minority reported having experienced a relapse $(23.9 \%)$. Subjects reported over 11 types of cancer diagnoses and cancer stages with breast cancer diagnosis being the highest rate $(35.8 \%)$. 


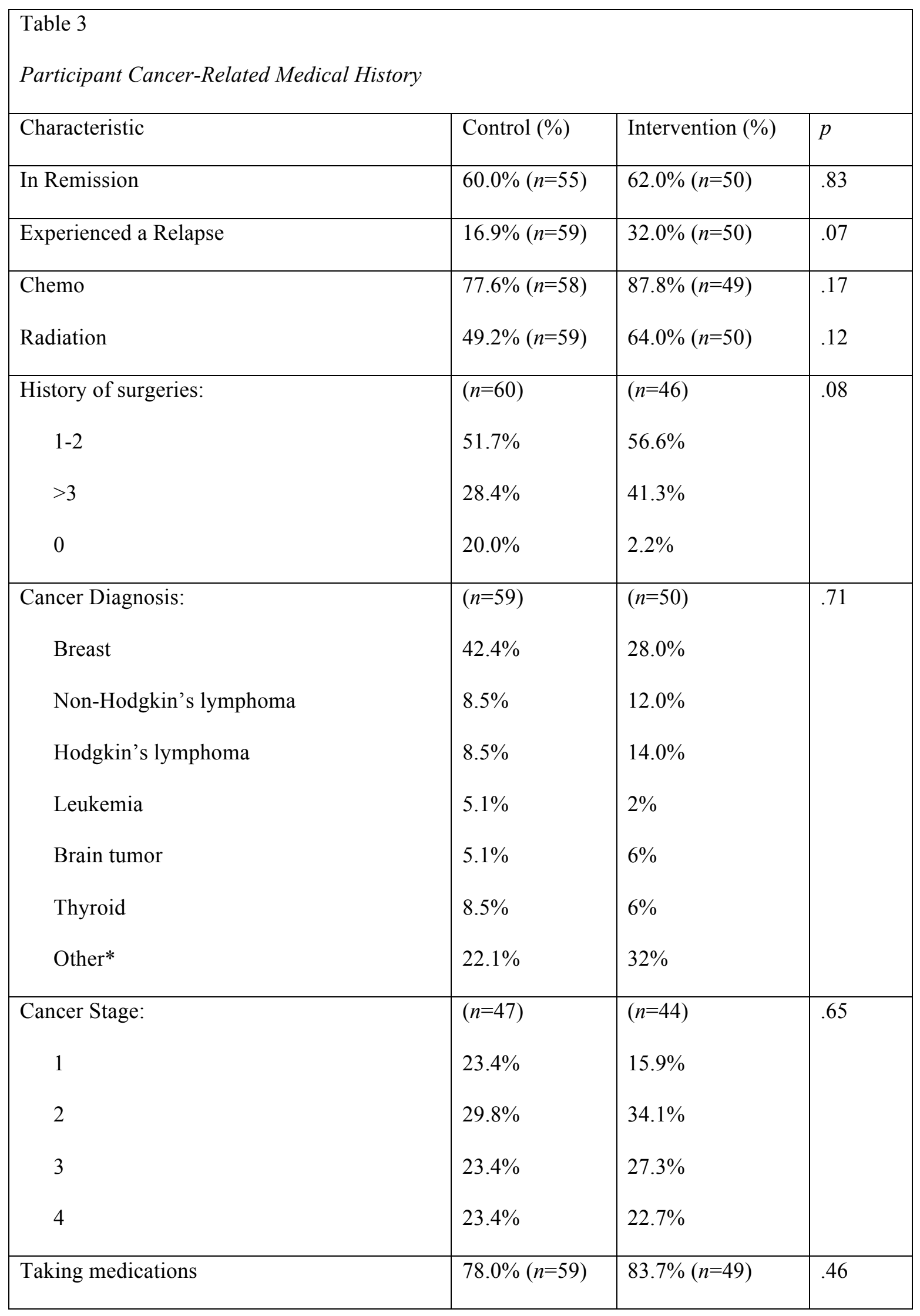


* Included Colon/Rectal, Ovarian, Testicular, Cervical, Gastric/Stomach, Bone, Carcinomas, Sarcomas including Ewing Sarcoma and Rhabdomyosarcoma, Renal Cell, Rectal, Mesothelioma, Melanoma, and Aplastic Anemia

\section{Retention At the 1 Week and 3 Month Assessments}

Retention at the 1 week (post-camp) assessment was high but significantly differed between groups ( $78.8 \%$ control vs. $100 \%$ intervention; $p=.001$ ). At 3 months, no significant differences in retention were observed $(66.7 \%$ control vs. $76 \%$ intervention; $p=.274)$. Examining differences in study completers vs. noncompleters at the 3 month assessment, noncompleters were more likely to be a non-smoker $(91.7 \%$ vs. $79 \% ; p=.033)$ or a current smoker $(4.2 \%$ vs. $0 \%$; $p=.033)$, or attending school $(35.7 \%$ vs. $13.8 \% ; p=.011)$. There were no significant differences between completers and noncompleters in other characteristics.

\section{Minutes of Physical Activity per Week}

Using intent to treat analysis, adjusting for covariates age, gender, age at diagnosis, and baseline minutes of PA/week, there was a significant difference $(p=.0001)$ in minutes of PA per week between intervention and control groups (Figure 1.1, Table 4). Bonferroni adjusted post-hoc analysis indicated that, relative to baseline, the intervention group had significantly $(\mathrm{p}=.0001)$ greater increases in PA at both 1 week (577 minutes vs. 9 minute increases) and 3 month followups (133 minute increases vs. 75 minute decreases; $p=.001$ ) respectively (Table 4). Exploratory analyses indicated no significant differences in weekly minutes of PA changes by type of camp $(p=.06)$. 


\begin{tabular}{|c|c|c|c|c|c|c|}
\hline $\begin{array}{l}\text { Table } 4 \\
\text { Intent to Treat } C \\
\text { Behavior }\end{array}$ & mpariso & of Camp vs. & tlist on Chang & in Physical Ac & ity and & edentary \\
\hline \multicolumn{2}{|c|}{ Subscale score } & Baseline & 1 Week & 3 Months & $\mathrm{F}$ & $p$ \\
\hline \multirow{2}{*}{$\begin{array}{l}\mathrm{PA} \\
\text { (minutes } \\
\mathrm{PA} / \text { week) }\end{array}$} & $\begin{array}{l}\mathrm{C} \\
(n=66)\end{array}$ & $374 \pm 341$ & $384 \pm 332$ & $299 \pm 299$ & \multirow[t]{2}{*}{51.57} & \multirow[t]{2}{*}{$.0001 *$} \\
\hline & $\begin{array}{l}\mathrm{I} \\
(n=49)\end{array}$ & $482 \pm 416$ & $1059 \pm 448$ & $615 \pm 449$ & & \\
\hline \multirow[t]{2}{*}{$\begin{array}{l}\text { Hours watching } \\
\text { TV (per week) }\end{array}$} & $\begin{array}{l}\mathrm{C} \\
(n=65)\end{array}$ & $10.35 \pm 8.67$ & $11.09 \pm 8.77$ & $13.86 \pm 15.14$ & \multirow[t]{2}{*}{7.24} & \multirow[t]{2}{*}{$.001 * *$} \\
\hline & $\begin{array}{l}\mathrm{I} \\
(n=51)\end{array}$ & $9.37 \pm 11.26$ & $2.25 \pm 4.23$ & $8.92 \pm 8.58$ & & \\
\hline \multirow{2}{*}{$\begin{array}{l}\text { Hours spent } \\
\text { sitting (per } \\
\text { week) }\end{array}$} & $\begin{array}{l}\mathrm{C} \\
(n=65)\end{array}$ & $30.77 \pm 23.03$ & $29.75 \pm 20.52$ & $28.03 \pm 21.07$ & \multirow[t]{2}{*}{11.37} & \multirow[t]{2}{*}{$.0001 * * *$} \\
\hline & $\begin{array}{l}\mathrm{I} \\
(n=51)\end{array}$ & $27.80 \pm 21.16$ & $11.16 \pm 9.23$ & $26.84 \pm 18.74$ & & \\
\hline
\end{tabular}

Note. Intent-to-treat analyses assumed baseline value for missing values. All analyses adjusted for baseline value, age, gender, and age at diagnosis. Unadjusted means presented in table for interpretive purposes. $C=$ control, $\mathrm{I}=$ intervention. $N$ 's vary due to differences in missing baseline data for dependent variables. Data represents means \pm standard deviations

* Post hoc analyses indicated significant difference in changes in minutes of PA per week from baseline to post-camp ( $p=.0001)$ and 3 months post-camp $(p=.001)$ between intervention and control groups. 
** Post hoc analyses indicated significant difference in changes in hours of TV watching per week from baseline to post-camp $(p=.001)$ between intervention and control groups. No differences at 3 months.

*** Post hoc analyses indicated significant changes in hours sitting per week from baseline to post-camp ( $p=.001)$ between intervention and control groups. No differences at 3 months. 
Figure 1.1

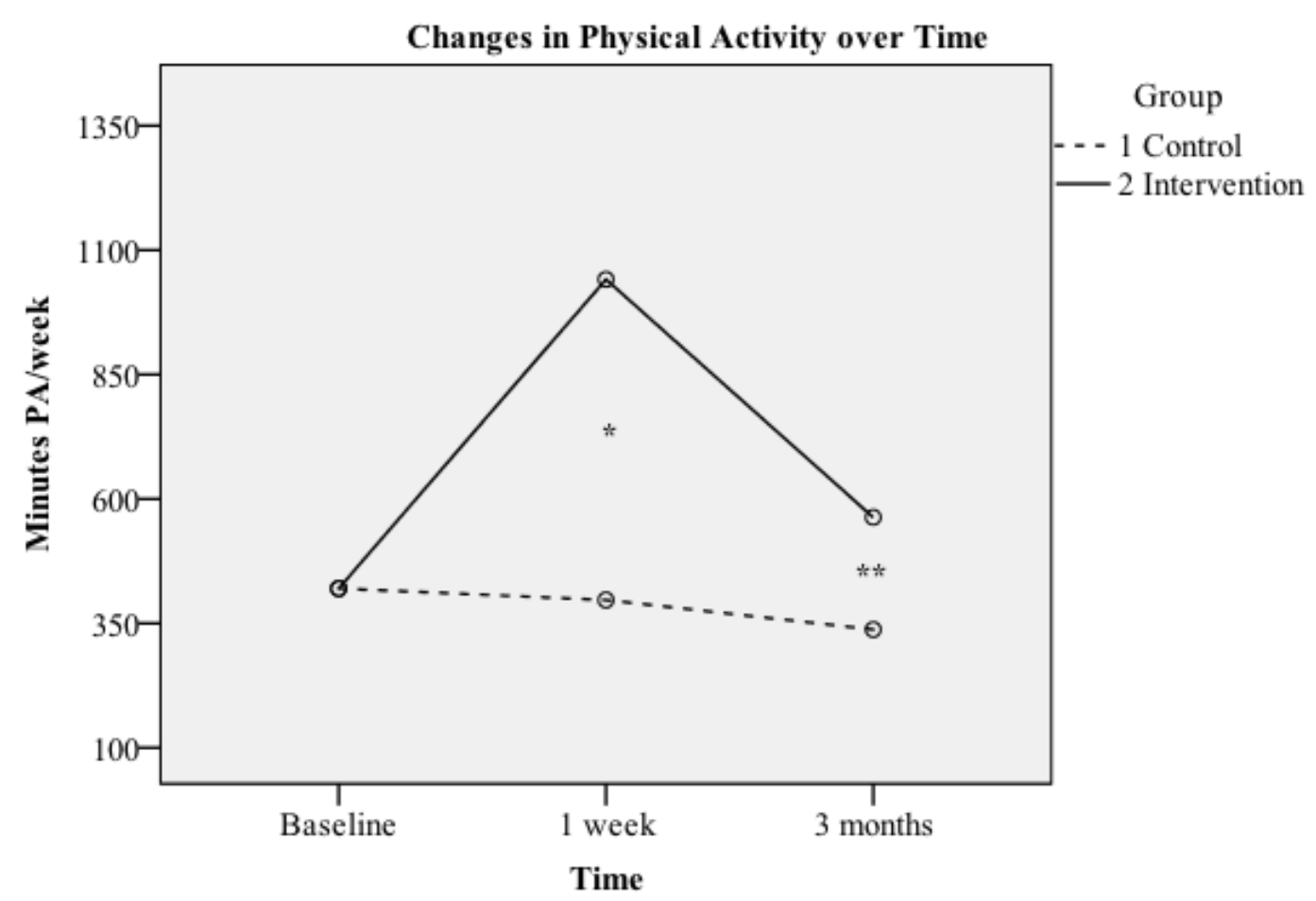

Figure 1.1. Changes in estimated marginal means of minutes of PA/week in the two groups. Group 1 = control, Group 2 = intervention. *Significant difference between $\mathrm{C}$ and $\mathrm{I}$ in post-hoc testing at 1 week $(\mathrm{p}=.0001) .{ }^{* *}$ Significant difference between $\mathrm{C}$ and $\mathrm{I}$ in post-hoc testing at 3 months $(\mathrm{p}=.001)$. 


\section{TV Viewing}

Using intent to treat analysis, adjusting for covariates, significant differences $(p=.001)$ in number of hours of TV viewing per week were observed between intervention and control groups (Figure 1.2, Table 4). Bonferroni adjusted post-hoc analysis indicated that the intervention group had significantly $(p=.001)$ greater reduction in hours of TV viewing per week than the control group at (7.1 reduction vs. 0.7 increase in TV hours/week, respectively) at the post-camp assessment. However, there was no significant difference $(p=.20)$ at 3 months. An exploratory partial correlation analysis adjusting for covariates revealed that changes in PA and TV viewing were not significantly correlated at any time point $(p=.801)$. 
Figure 1.2

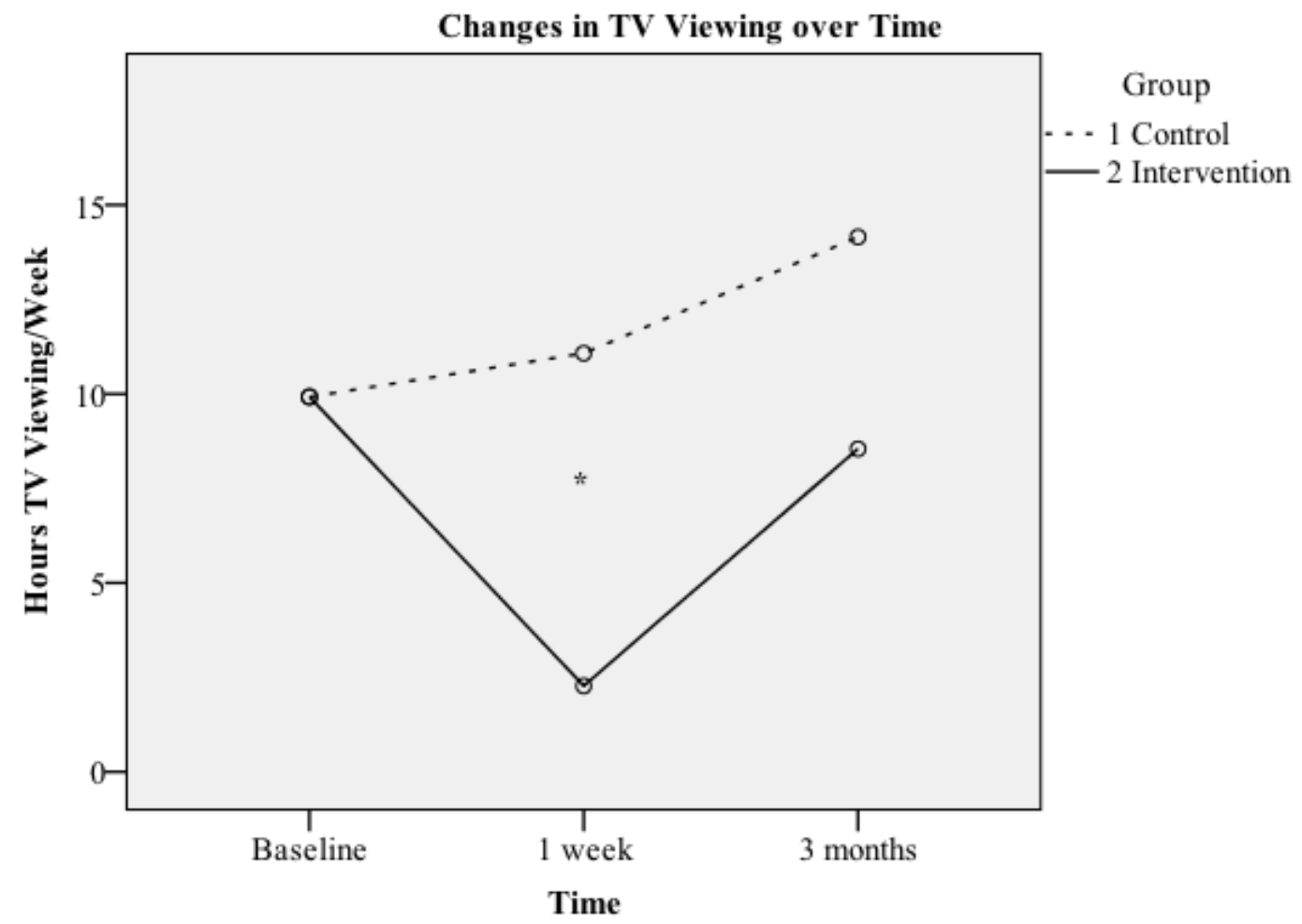

Figure 1.2 Changes in Estimated Marginal Means of Hours TV Viewing/Week in the Two Groups. Group 1 = control, Group 2 = intervention. *Significant difference bwtween $\mathrm{C}$ and $\mathrm{I}$ in post-hoc testing at 1 week $(\mathrm{p}=.001)$ 


\section{Hours Sitting}

Using intent to treat analysis, adjusting for covariates, there was a significant difference in changes in hours sitting per week between intervention and control groups (Figure 1.3, Table 4). Bonferroni adjusted post-hoc analysis indicated that the intervention group had significantly ( $p=.001)$ fewer hours of sitting per week than the control group at the end of camp (16.7 decrease in hours sitting/week vs. 1 decrease in hours sitting/week, respectively). However, there was no

significant difference $(p=.75)$ between changes in hours sitting between intervention and control at 3 months. An exploratory partial correlation analysis adjusting for covariates revealed that changes in PA and hours sitting were not significantly correlated at any time point $(p=.66)$. 
Figure 1.3

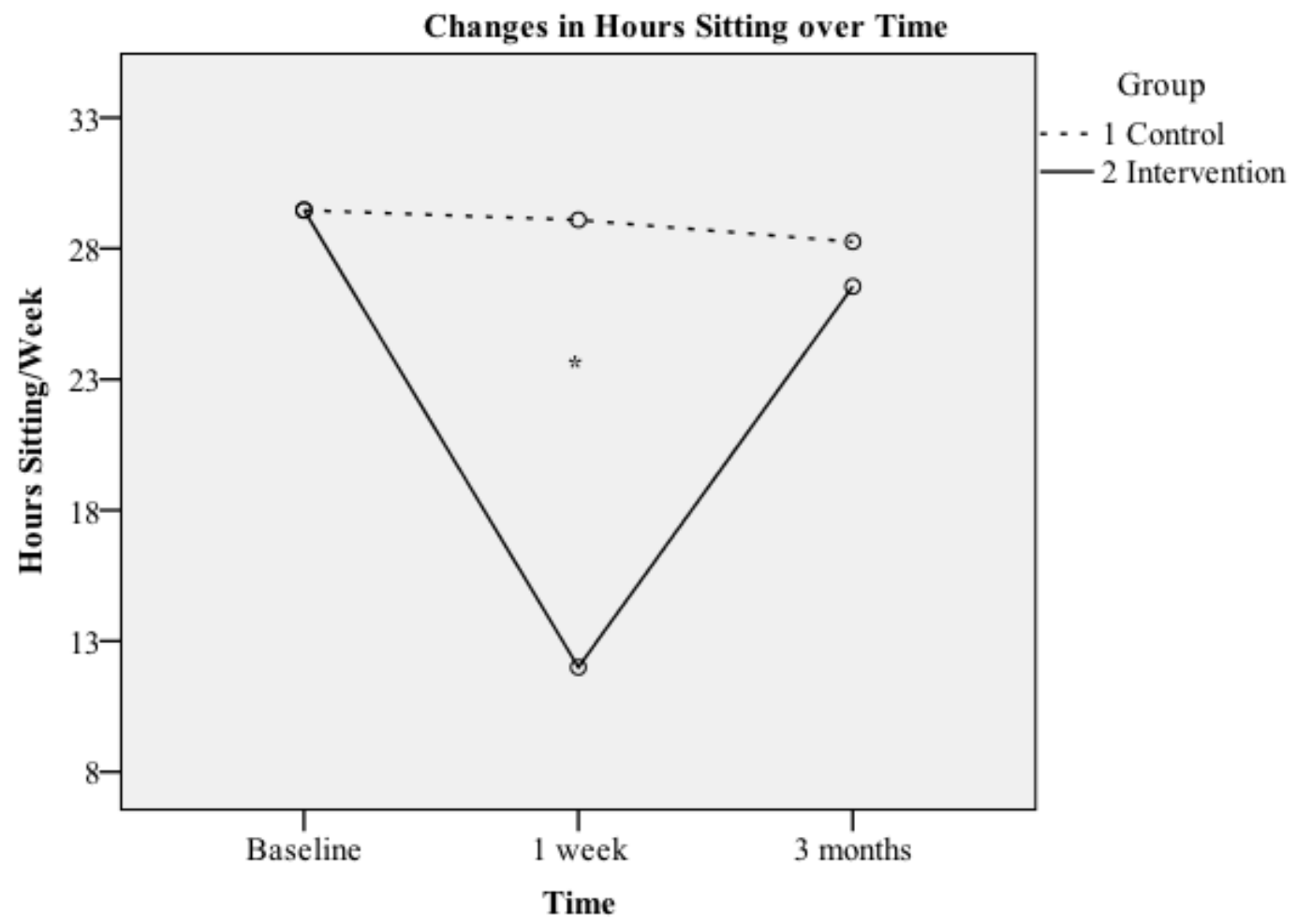

Figure 1.3 Changes in Estimated Marginal Means of Hours Sitting/Week in the Two Groups. Group $1=$ control, Group 2 = intervention. *Significant difference between I and $\mathrm{C}$ in post-hoc testing at 1 week $(\mathrm{p}=.001)$ 


\section{Exercise Self-Efficacy}

Using intent to treat analysis, adjusting for covariates, there were no significant differences in changes in the two exercise self-efficacy subscale scores: sticking to it $(p=.29)$ or making time for exercise $(p=.34)$ between intervention and control groups (Table 5). 


\begin{tabular}{|c|c|c|c|c|c|c|}
\hline \multicolumn{7}{|c|}{$\begin{array}{l}\text { Table } 5 \\
\text { Intent to Treat Comparison of Camp vs. Waitli } \\
\text { Environmental Change Self-Efficacy }\end{array}$} \\
\hline \multicolumn{2}{|c|}{ Subscale score } & Baseline & 1 Week & 3 Months & $\mathrm{F}$ & $p$ \\
\hline \multirow[t]{2}{*}{ Sticking to it } & $\mathrm{C}(n=54)$ & $3.64 \pm .80$ & $3.60 \pm .87$ & $3.61 \pm .96$ & \multirow[t]{2}{*}{1.25} & \multirow[t]{2}{*}{.29} \\
\hline & $\mathrm{I}(n=39)$ & $3.75 \pm .82$ & $3.80 \pm .74$ & $3.80 \pm . .81$ & & \\
\hline \multirow{2}{*}{$\begin{array}{l}\text { Making time for } \\
\text { exercise }\end{array}$} & $\mathrm{C}(n=56)$ & $3.81 \pm .78$ & $3.71 \pm .86$ & $3.70 \pm .97$ & \multirow[t]{2}{*}{1.08} & \multirow[t]{2}{*}{.34} \\
\hline & $\mathrm{I}(n=42)$ & $4.01 \pm .66$ & $3.94 \pm .74$ & $4.04 \pm .75$ & & \\
\hline \multirow{2}{*}{$\begin{array}{l}\text { Finding social } \\
\text { environment }\end{array}$} & $\mathrm{C}(n=66)$ & $2.14 \pm 1.00$ & $2.11 \pm .88$ & $2.13 \pm .93$ & \multirow[t]{2}{*}{1.80} & \multirow[t]{2}{*}{.17} \\
\hline & $\mathrm{I}(n=50)$ & $2.45 \pm .88$ & $2.62 \pm .93$ & $2.44 \pm .95$ & & \\
\hline \multirow{2}{*}{$\begin{array}{l}\text { Finding physical } \\
\text { environment }\end{array}$} & $\mathrm{C}(n=66)$ & $2.73 \pm .94$ & $2.81 \pm .97$ & $2.71 \pm .90$ & \multirow[t]{2}{*}{1.69} & \multirow[t]{2}{*}{.19} \\
\hline & $\mathrm{I}(n=50)$ & $3.17 \pm .78$ & $3.34 \pm .68$ & $3.09 \pm .75$ & & \\
\hline \multirow{2}{*}{$\begin{array}{l}\text { Creating physical } \\
\text { environment }\end{array}$} & $\mathrm{C}(n=66)$ & $1.06 \pm 1.14$ & $1.30 \pm 1.24$ & $1.26 \pm 1.10$ & \multirow[t]{2}{*}{.13} & \multirow[t]{2}{*}{.88} \\
\hline & $\mathrm{I}(n=50)$ & $1.37 \pm 1.16$ & $1.62 \pm 1.24$ & $1.54 \pm 1.19$ & & \\
\hline
\end{tabular}

Note. Intent-to-treat analyses assumed baseline value for missing values. All analyses adjusted for baseline value, age, gender, and age at diagnosis. Unadjusted means presented in table for interpretive purposes. $\mathrm{C}=$ control, $\mathrm{I}=$ intervention. $N$ 's vary due to differences in missing baseline data for dependent variables. Data represents means \pm standard deviations 


\section{Environmental Change Self-Efficacy}

As shown in Table 5, using intent to treat analysis, adjusting for covariates, there were no significant differences in changes in any of the environmental change self-efficacy scores between intervention and control groups (Table 5): finding social environment $(p=.17)$, finding physical environment $(p=.19)$, or creating physical environment $(p=.88)$.

\section{Perceived Barriers to Physical Activity}

Using intent to treat analysis, adjusting for covariates, there were significant group differences $(p=.04)$ in the excuses score for perceived barriers to PA questionnaire. (Table 6). Bonferroni adjusted post-hoc analysis indicated the intervention group had significantly $(p=.007)$ greater reduction in Excuses subscale score at the end of camp (.21 decrease vs. .03 increase in subscale score). However, there was no significant difference in changes in excuses scores at 3 months $(p=.95)$. There were also no significant differences in the other perceived barriers to exercise scores for aversiveness $(p=.24)$, inconvenience (.92), worries $(p=.14)$, demands $(p=.64)$, or average barriers score $(p=.23)$ between the intervention and control groups. 


\begin{tabular}{|c|c|c|c|c|c|c|}
\hline \multicolumn{7}{|c|}{$\begin{array}{l}\text { Table } 6 \\
\text { Intent to Treat Comparison of Camp vs. Waitlist Control on Changes in Perceived Barriers to } \\
\text { Physical Activity }\end{array}$} \\
\hline \multicolumn{2}{|c|}{ Subscale score } & \multirow{2}{*}{$\begin{array}{l}\text { Baseline } \\
.90 \pm .65\end{array}$} & \multirow{2}{*}{$\begin{array}{c}1 \text { Week } \\
1.01 \pm .73\end{array}$} & \multirow{2}{*}{$\begin{array}{l}3 \text { Months } \\
.90 \pm .73\end{array}$} & \multirow{2}{*}{$\begin{array}{r}\mathrm{F} \\
1.46\end{array}$} & \multirow{2}{*}{$\begin{array}{r}p \\
.24\end{array}$} \\
\hline Aversiveness & $\mathrm{C}(n=65)$ & & & & & \\
\hline & $\mathrm{I}(n=50)$ & $.94 \pm .69$ & $.96 \pm .69$ & $.99 \pm .73$ & & \\
\hline \multirow[t]{2}{*}{ Inconvenience } & $\mathrm{C}(n=65)$ & $1.16 \pm .68$ & $1.20 \pm .80$ & $1.13 \pm .74$ & \multirow[t]{2}{*}{.088} & \multirow[t]{2}{*}{.92} \\
\hline & $\mathrm{I}(n=50)$ & $1.00 \pm .65$ & $1.03 \pm .65$ & $1.00 \pm .68$ & & \\
\hline \multirow[t]{2}{*}{ Worries } & $\mathrm{C}(n=65)$ & $.79 \pm .63$ & $.84 \pm .72$ & $.73 \pm .62$ & \multirow[t]{2}{*}{1.96} & \multirow[t]{2}{*}{.14} \\
\hline & $\mathrm{I}(n=50)$ & $.88 \pm .69$ & $.77 \pm .63$ & $.80 \pm .69$ & & \\
\hline \multirow[t]{2}{*}{ Excuses } & $\mathrm{C}(n=65)$ & $2.08 \pm .68$ & $2.11 \pm .72$ & $2.05 \pm .78$ & \multirow[t]{2}{*}{3.42} & \multirow[t]{2}{*}{.04} \\
\hline & I $(n=49)$ & $2.01 \pm .74$ & $1.79 \pm .65$ & $1.96 \pm .84$ & & \\
\hline \multirow[t]{2}{*}{ Demands } & $\mathrm{C}(n=66)$ & $1.36 \pm .93$ & $1.53 \pm .87$ & $1.49 \pm .84$ & \multirow[t]{2}{*}{.45} & \multirow[t]{2}{*}{.64} \\
\hline & $\mathrm{I}(n=50)$ & $1.38 \pm .83$ & $1.46 \pm .78$ & $1.37 \pm .84$ & & \\
\hline \multirow[t]{2}{*}{ Barriers average } & $\mathrm{C}(n=62)$ & $1.22 \pm .49$ & $1.29 \pm .57$ & $1.22 \pm .58$ & \multirow[t]{2}{*}{1.48} & \multirow[t]{2}{*}{.23} \\
\hline & $\mathrm{I}(n=49)$ & $1.18 \pm .53$ & $1.15 \pm .52$ & $1.17 \pm .58$ & & \\
\hline
\end{tabular}

Note. Intent-to-treat analyses assumed baseline value for missing values. All analyses adjusted for baseline value, age, gender, and age at diagnosis. Unadjusted means presented in table for interpretive purposes. $C=$ control, $\mathrm{I}=$ intervention. $N$ 's vary due to differences in missing baseline data for dependent variables. Data represents means \pm standard deviations

* Post hoc analyses indicated significant changes in Perceived Barriers to PA "Excuses "score from baseline to post-camp $(p=.007)$ between intervention and control groups. No differences at 3 months. 


\section{Physical Activity Variety}

Using intent to treat analysis, adjusting for covariates, there was a significant difference $(p=.0001)$ in PA variety per week between intervention and control groups (Table 7). Bonferroni adjusted post-hoc analysis indicated that the intervention group had significantly $(p=.0001)$ greater increases in PA variety at end of camp (1.35 vs. -.06 increases in number of different types of activities/week). However, there was no significant difference $(p=.700)$ in changes in PA variety between intervention and control at the 3 month follow-up (Table 7). 


\begin{tabular}{|c|c|c|c|c|c|c|}
\hline $\begin{array}{l}\text { Table } 7 \\
\text { Intent to Treat Co }\end{array}$ & arison of $C$ & amp vs. Wai & ist on Chang & in PA Vari & and $E r$ & ment of \\
\hline \multicolumn{2}{|c|}{ Subscale score } & Baseline & 1 Week & 3 Months & $\mathrm{F}$ & $p$ \\
\hline \multirow{2}{*}{$\begin{array}{l}\text { PA variety } \\
\text { (activities/week) }\end{array}$} & $\mathrm{C}(n=65)$ & $2.34 \pm 1.15$ & $2.28 \pm 1.05$ & $2.23 \pm 1.14$ & \multirow[t]{2}{*}{33.52} & \multirow[t]{2}{*}{$.0001^{*}$} \\
\hline & $\mathrm{I}(n=49)$ & $2.80 \pm 1.34$ & $4.14 \pm 1.61$ & $2.78 \pm 1.28$ & & \\
\hline \multirow{2}{*}{$\begin{array}{l}\text { Structured } \\
\text { activities }\end{array}$} & $\mathrm{C}(n=66)$ & $3.16 \pm .73$ & $3.25 \pm .69$ & $3.12 \pm .71$ & \multirow[t]{2}{*}{3.167} & \multirow[t]{2}{*}{$.04 * *$} \\
\hline & $\mathrm{I}(n=49)$ & $3.35 \pm .59$ & $3.40 \pm .58$ & $3.43 \pm .65$ & & \\
\hline \multirow{2}{*}{$\begin{array}{l}\text { Unstructured } \\
\text { activities }\end{array}$} & $\mathrm{C}(n=66)$ & $2.60 \pm 1.09$ & $2.75 \pm 1.06$ & $2.75 \pm 1.14$ & \multirow[t]{2}{*}{.316} & \multirow[t]{2}{*}{.73} \\
\hline & $\mathrm{I}(n=50)$ & $2.49 \pm .86$ & $2.60 \pm .79$ & $2.67 \pm .97$ & & \\
\hline \multirow[t]{2}{*}{ Walking } & $\mathrm{C}(n=66)$ & $3.92 \pm 1.11$ & $4.08 \pm 1.04$ & $3.88 \pm 1.16$ & \multirow[t]{2}{*}{2.12} & \multirow[t]{2}{*}{.12} \\
\hline & $\mathrm{I}(n=50)$ & $4.02 \pm 1.06$ & $4.18 \pm .94$ & $4.16 \pm .93$ & & \\
\hline
\end{tabular}

Note. Intent-to-treat analyses assumed baseline value for missing values. All analyses adjusted for baseline value, age, gender, and age at diagnosis. Unadjusted means presented in table for interpretive purposes. $\mathrm{C}=$ control, $\mathrm{I}=$ intervention. $\mathrm{N}$ 's vary due to differences in missing baseline data for dependent variables. Data represents means \pm standard deviations.

* Post hoc analyses indicated significant difference in changes in PA Variety per week from baseline to post-camp ( $\mathrm{p}=.0001)$ between intervention and control groups. No differences at 3 months.

**Changes in Enjoyment of Structured Activities from baseline. Post-hoc tests were not significant. 


\section{Enjoyment of Physical Activity}

Using intent to treat analysis, adjusting for covariates, there was a significant difference $(p=.04)$ in Enjoyment of Structured Activities, suggesting greater increases in enjoyment in intervention than control groups (Table 7). However, Bonferonni adjusted post-hoc tests were not significant at 1 week $(p=.11)$ and 3 months $(p=.31)$. There were also no significant differences in Enjoyment of Unstructured Activities $(p=.73)$ or Walking $(p=.12)$ at end of camp or 3 months follow-up between the intervention and control groups.

Logistic regression analysis adjusting for covariates examined the relationship between group status and high vs. low preference for Vigorous PA at end of camp and at the 3 month follow-up (Table 8). No significant differences were observed at either period $(p=.76 ; p=.58$, respectively). Similar analysis was done comparing high versus low preference for Moderate PA. No significant differences were observed at end of camp or 3 month follow-up $(p=.34 ; p=.47$, respectively). Moreover, comparing high versus low preference for Inactive Recreation, no significant differences were observed at end of camp $(p=.97)$. For the analysis at 3 month followup, analysis was unable to be performed as only 3 participants had a high preference for inactive recreation at the 3 month follow-up. 
Table 8

Intent to Treat Comparison of Camp vs. Waitlist on Changes in High or Low Preference for

Vigorous, Moderate, or Inactive Recreation

\begin{tabular}{|l|l|l|l|l|l|l|}
\hline \multirow{2}{*}{ PA } & \multicolumn{2}{|l|}{ Vigorous } & \multicolumn{2}{l|}{ Moderate } & \multicolumn{2}{l|}{ Inactive Recreation } \\
\cline { 2 - 7 } & 1 Week & 3 Month & 1 Week & 3 Month & 1 Week & 3 Month \\
\hline Group & $1.21[.37$, & $1.36[.45$, & $.52[.14$, & $.65[.19$, & $1.06[.07$, & NS \\
& $3.97]$ & $4.13]$ & $1.99]$ & $2.14]$ & $15.71]$ & \\
& $p=.76$ & $p=.58$ & $p=.34$ & $p=.47$ & $p=.97$ & \\
& & & & & & \\
\hline
\end{tabular}

Note. Intent-to-treat analyses assumed baseline value for missing values. All analyses adjusted

for baseline value, age, gender, and age at diagnosis. Unadjusted means presented in table for interpretive purposes. Data represent 95\% confidence intervals for PA Preferences. 


\section{Chapter 5}

\section{Discussion}

The primary purpose of this study was to determine whether a week-long outdoor adventure therapy program for YACS would increase PA levels. The secondary purpose was to determine if the camp increased key correlates of PA, including exercise self-efficacy, environmental change self-efficacy, PA enjoyment, and PA variety, and reduce sedentary behavior and perceived barriers to exercise. The main findings of this study supported the hypothesis that the camp increased PA levels and decreased sedentary behaviors and barriers; however, the impacts on correlates of PA were less substantial.

As noted, the week-long outdoor adventure therapy camp had a significant effect on increasing PA levels both at 1 week and the 3 month follow-up. Other studies that have reported positive effects on increasing PA utilized interventions that were much longer in duration, such as an 8-week aerobic based group exercise program for older cancer survivors or a 6-month homebased exercise intervention (Broderick et al., 2014; Basen-Enquist et al., 2013). This study was the first to show that a week long intervention had a significant effect on increasing PA through 3 months of follow-up. These findings suggested potential efficacy of outdoor adventure therapy as an alternative method to increase PA in YACS. While levels of PA increased from baseline to 1 week, these were attenuated (although still above baseline) at 3 months, suggesting a weakening effect over time. Similar findings were observed in a 12-week home-based PA intervention in older colorectal cancer survivors where significant differences between intervention and control were found at 12 weeks, but attenuated at 6 and 12 months (Pinto et al., 2013). Future research should examine methods to improve long-term effects after camp termination, such as whether booster camps throughout the year could help maintain a lasting effect on increasing PA in young adult cancer survivors.

The outdoor adventure therapy camp intervention group also appeared to result in decreases in sedentary behavior, measured by hours of TV viewing and hours spent sitting per 
week, at 1 week but not 3 months post-camp. The reduction in sedentary behavior was possibly a result of the camp agenda in which participants had little time for sedentary activities (e.g., activities throughout the day, community meals, campfires in the evening). The decrease in TV Viewing hours was also possibly a result of a lack of TVs present at the participant and staff housing during the camp. It's possible that the return to participants' daily life increased exposure to TVs at home and in social or work situations. Future research should examine whether removing TVs from the home or work environment could result in maintaining a decrease in TV Viewing hours/week post-camp. Of note, the levels of TV Viewing at baseline and 3 month follow-up for both intervention and control group (Table 4) were 8-10 hours per week. This is of concern since epidemiological research has shown that individuals who report over 7 hours TV Viewing/week (as compared to those that watched less than 1 hour/day) were at greater risk for all-cause mortality, cardiovascular disease, and cancer mortality, as found in a study of 240,819 healthy adults followed for 8.5 years after adjusting for MVPA (Matthews et al., 2012).

Due to the benefits of decreasing sedentary behavior in cancer survivors, an outdoor adventure therapy program that addresses reducing sedentary behavior or TV viewing might improve long-term health outcomes. Of note, TV Viewing and PA were not correlated, suggesting that increase in PA does not necessarily displace TV Viewing. Other studies have demonstrated this lack of association in non-cancer populations (Vandelanotte et al., 2009). Examining barriers to exercise, the camp had a significant effect on reducing "Excuses" for not exercising during camp, but not at the 3 month follow-up. Participants were less likely to report feelings of being too tired to exercise or having a lack of time, energy, or self-discipline or willpower to exercise (questions included in the Excuses subscale) at 1 week but not 3 months. The 1 week effect demonstrates the immediate impact of camp on reducing excuses and other barriers to performing exercise. Other research has shown immediate and longer term positive impacts of PA interventions on barriers. In a RCT of 41 breast cancer survivors, the 3-month SCT based intervention resulted in lower perceived barriers as compared with the usual care control 
group; barriers interference accounted for $39 \%$ of the intervention effect on PA at 3 months postintervention Rogers et al. (2011). This successful intervention specifically targeted barriers to PA and self-efficacy during individual sessions with participants over 3 months. Future research should examine whether targeting barriers and PA during camp could produce sustained improvements in perceived barriers through 3 months following the camp, as well as potentially reduce other types of perceived barriers to exercise that did not appear to change significantly during camp (aversiveness, inconvenience, worries, demands, or average barriers score).

The outdoor adventure therapy camp also had a significant effect on increasing PA variety, or the number of types of physical activities engaged in each week. To no surprise, PA variety increased during the camp but went back to baseline levels after participants returned home. The outdoor adventure therapy camp model might serve as an effective way of exposing YACS to different possible types of physical activities. It is possible that effects of increasing PA variety were not maintained at 3 months because the types of outdoor adventure activities were not accessible to the participants once they returned home. In obesity interventions, PA variety has been linked with greater long-term weight control and higher levels of activity overall (Bond et al., 2012; Raynor, Bond, Steeves, and Thompson (2014). However, relatively few studies have examined this in YACS. Future research should examine ways to make a variety of activities accessible once these young adult participants return home.

Intervention participants reported greater increases in enjoyment of structured PA over time (such as team sports, cycling, jogging, swimming and dancing) in comparison with the control group. These findings are consistent with results from a study of 21 breast cancer survivors undergoing treatment in which a higher daily energy expenditure (kilocalories per kilogram body weight per day) was significantly associated with, among other correlates, higher PA enjoyment (Rogers et al., 2005). However, there were no significant effects on increasing enjoyment of unstructured PA such as digging in the garden, raking leaves, etc. These results suggest that the nature of the outdoor adventure therapy program increased enjoyment of PA, 
perhaps through its emphasis on competition component. Also, the structured activities in the outdoor adventure therapy camp might be more enjoyable as participants have opportunities to compete and bond towards achieving a goal or challenge that is also rewarding and enjoyable (i.e., reaching the top of a rock climb; successfully kayaking a challenging rapid). The outdoor adventure therapy camp model is designed to challenge participants to push their limits physically and mentally, which may have increased enjoyment of structured activities.

Although enjoyment of structured activities increased, there was no significant effect of the intervention on changes in preferences for vigorous, moderate, or inactive recreation activities in comparison with the control group. Overall, both groups reported greater preference for vigorous vs. moderate PA. It is possible that people who are motivated to enroll in the outdoor adventure therapy camp have higher preferences for vigorous activity in general. Also, the weeklong camp might not be long enough to alter preferences.

The psychosocial mechanisms behind the increase in PA remain unclear. Surprisingly, in contrast with the hypothesis, there was no significant effect of the camp experience on exercise self-efficacy. These findings are in contrast with studies that have found self-efficacy to be associated with PA interventions in cancer survivors. After a 1 year intervention in older breast cancer survivors, participants with higher self-efficacy reported higher levels of PA at the 6month follow up (Loprinzi et al., 2012). Another PA intervention framed on TTM and SCT in breast cancer survivors found that exercise self-efficacy significantly predicted exercise adherence (Pinto et al., 2009). In the current study, there might not have been enough time to build self-efficacy in the week-long camp. In addition, there was no formal intervention utilized in the camp to increase self-efficacy during or after the camp. Another potential reason for the lack of change in self-efficacy could be that the measures used did not capture the type of selfefficacy that would increase from an outdoor adventure camp. The exercise self-efficacy scale used has been validated in healthy individuals, but has not been used in studies examining PA interventions in cancer survivors (Sallis et al., 1987). 
There was also no significant effect of the camp experience on environmental change self-efficacy. The environmental change self-efficacy scale was a new measure in which preliminary findings from a sample of college students indicated that the scales had acceptable test-test reliability and factorial validity, but it had never been studied with cancer survivors (H. Starnes, personal communication, February 28, 2015). The questionnaire asked participants, how confident they are in being able to find and create physical and social environments to be physically active such as "confidence in finding coworkers/classmates to be physically active with, finding a park near their home where they can be physically active, or asking community leaders to create places to be physically active." It is perhaps no surprise that the answers did not change at the end of camp, since participants were not back home yet and didn't yet have the opportunity to change their confidence in finding ways to be physically active at home. In addition, the outdoor adventure camp did not specifically target methods to find and create physical and social environments for PA once the participants returned home. This is perhaps another area that could be integrated in the camp to improve its long-term effects.

It is possible that the outdoor adventure therapy camp increased other, unmeasured psychosocial aspects related to changes in PA levels. A recent study published on the outdoor adventure therapy organization First Descents demonstrated that compared with a control group, first time camp participants had improvements in body image, self-compassion and self-esteem, and less depression and alienation relative to pre-test (Rosenberg, Lange, Zebrack, Moulton, \& Kosslyn, 2014). However, no other research has been performed on the organization and limited quantitative studies exist on the benefits of outdoor adventure therapy for YACS. Further research is needed to determine the relationship between PA and its correlates in outdoor adventure therapy interventions for cancer survivors.

Both control and intervention groups also had a relatively high average level of PA per week at baseline (374 minutes vs. 482 minutes, respectively). It's possible that the type of people 
who choose to apply for an outdoor adventure therapy camp are, on average, more physically active than the general population of YACS and/or more likely to meet PA recommendation of 150 minutes of moderate PA per week (Schmitz et al., 2010). Future analysis will examine proportions of participants from the intervention and control group who met recommended PA guidelines across time. Nonetheless, the high level of activity at entry into the camp could have resulted in a "ceiling effect" with regards to the magnitude of influence of an outdoor adventure camp on improving PA levels and correlates of PA..

One strength of the study is that it was the first quantitative study of the effects of an outdoor adventure therapy program on increasing PA of YACS in comparison with a control group at end of camp, as well as 3 months following the intervention. Furthermore, it was among the first studies that examined methods to increase PA in YACS. This study utilized an already existing outdoor adventure therapy program; its short-term, 1-week duration might be beneficial for cancer survivors with busy schedules or other work, family, or time commitments that might prevent them from enrolling in a longer intervention. Other strengths included a prospective study following a male and female population with a variety of different cancer diagnoses and cancer stages with a similarly matched control group. This study used validated quantitative questionnaires to obtain self-reported data, including the 7-Day PAR, which is an extensive and thorough method of obtaining information on PA levels (Sallis et al., 1985). Another strength of the study is that it examined the possible correlates theorized to increase PA levels. This study was also a co-study of a qualitative study measuring program outcomes of participation in First Descents, and this quantitative portion of the study could be used for triangulation purposes for the qualitative study.

The limitations of this study include a non-randomized design, which limited the extent to how changes could be definitively attributed to the camp. This study also had a risk of selection bias, as participants in the study elected to apply to attend a First Descents camp. 
Also, while not significantly different, the intervention group had a greater percentage of participants who experienced a relapse or received chemotherapy and radiation therapy, which could reflect selection bias on the part of the organization's selection of camp vs. waitlist participants. Future research should examine if adjusting for relapse status and history of chemotherapy and radiation therapy influenced outcomes. Participants' preference for camp location and modality was unmeasured, and participants may not have received their first choice due to camp size limitations, which may have influenced outcomes of participation. Other limitations included a lack of external validity to YACS participating in other types of exercise interventions. There was a loss of participants at follow-up, which again limited generalizability. Intent to treat analyses were conducted, but it remains possible that participants who were absent had deterioration in behaviors above baseline. Furthermore, data were collected based on selfreport, which has validity limitations in comparison to objective measures (Sallis and Saelens, 2000).

Findings from this study suggested that outdoor adventure therapy may have been an effective method for increasing PA in YACS. Nonetheless, effects tended to wane after camp termination. Future research should examine ways to increase PA after camp termination and maintain benefits observed during camp of increasing PA variety, enjoyment of PA, and reduced barriers and sedentary behavior. Possible methods of maintaining long-term benefits, such as such as attending more camps throughout the year, or removing TVs from the home or work environment, should be examined. It's also possible that an outdoor adventure therapy intervention that incorporates and educates participants on the benefits of PA for cancer survivors, such as reducing reoccurrence of cancer, prolonging lifespan, and alleviating symptoms of treatment, might serve as a motivating factor to increase PA levels long-term. Future randomized controlled trials are needed with larger sample sizes and a greater variety of participants in terms of gender and ethnic backgrounds. Incorporating more follow-up measurements after returning home from camp up to 1 year later might provide greater insight 
into the optimal timing for interventions to maintain improvements in PA post camp. In addition, a variety of outdoor adventure therapy programs for young adult cancer survivors should be examined, including programs with an intervention component designed to increase PA and correlates of PA such as self-efficacy. 


\section{Bibliography}

Bandura, A. (1977). Self-efficacy: toward a unifying theory of behavioral change. Psychological Review, 84(2), 191-215.

Bandura, A. (2004). Health promotion by social cognitive means. Health Education \& Behavior, 31(2), 143-164. doi: 10.1177/1090198104263660

Basen-Engquist, K., Carmack, C. L., Li, Y., Brown, J., Jhingran, A., Hughes, D. C., . . Waters, A. (2013). Social-cognitive theory predictors of exercise behavior in endometrial cancer survivors. Health Psychology, 32(11), 1137-1148. doi: 10.1037/a0031712

Bélanger, L. J., Plotnikoff, R. C., Clark, A., \& Courneya, K. S. (2011). Physical activity and health-related quality of life in young adult cancer survivors: a Canadian provincial survey. Journal of Cancer Survivorship, 5(1), 44-53. doi: 10.1007/s11764-010-0146-6

Bond, D. S., Raynor, H. A., Phelan, S., Steeves, J., Daniello, R., \& Wing, R. R. (2012). The Relationship between Physical Activity Variety and Objectively Measured Moderate-toVigorous Physical Activity Levels in Weight Loss Maintainers and Normal-Weight Individuals. Journal of Obesity, 2012, 812414. doi: 10.1155/2012/812414

Broderick, J. M., Guinan, E., O' Donnell, D. M., Hussey, J., Tyrrell, E., \& Normand, C. (2014). Calculating the costs of an 8-week, physiotherapy-led exercise intervention in deconditioned cancer survivors in the early survivorship period (the PEACH trial). Physiotherapy, 100(2), 182-184. doi: 10.1016/j.physio.2013.12.003

Calfas, K. J., Sallis, J. F., Nichols, J. F., Sarkin, J. A., Johnson, M. F., Caparosa, S., . . . Alcaraz, J. E. (2000). Project GRAD: two-year outcomes of a randomized controlled physical activity intervention among young adults. Graduate Ready for Activity Daily. American Journal of Preventive Medicine, 18(1), 28-37.

Carter, C. L., Onicescu, G., Cartmell, K. B., Sterba, K. R., Tomsic, J., \& Alberg, A. J. (2012). The comparative effectiveness of a team-based versus group-based physical activity 
intervention for cancer survivors. Supportive Care in Cancer, 20(8), 1699-1707. doi: $10.1007 / \mathrm{s} 00520-011-1263-0$

Doyle, C., Kushi, L. H., Byers, T., Courneya, K. S., Demark-Wahnefried, W., Grant, B., . . . Society, A. C. (2006). Nutrition and physical activity during and after cancer treatment: an American Cancer Society guide for informed choices. CA: A Cancer Journal for Clinicians, 56(6), 323-353.

Epstein, I. (2004). Adventure therapy: a mental health promotion strategy in pediatric oncology. Journal of Pediatric Oncology Nursing, 21(2), 103-110.

First Descents. 2014 About Us. FirstDescents.Org. Retrieved from http://firstdescents.org/about-us

Fotheringham, M. J., Wonnacott, R. L., \& Owen, N. (2000). Computer use and physical inactivity in young adults: public health perils and potentials of new information technologies. Annals of Behavioral Medicine, 22(4), 269-275.

Groll, D. L., To, T., Bombardier, C., \& Wright, J. G. (2005). The development of a comorbidity index with physical function as the outcome. Journal of Clinical Epidemiology, 58(6), 595-602. doi: 10.1016/j.jclinepi.2004.10.018

Hocking, M. C., Schwartz, L. A., Hobbie, W. L., Derosa, B. W., Ittenbach, R. F., Mao, J. J., . . Kazak, A. E. (2013). Prospectively examining physical activity in young adult survivors of childhood cancer and healthy controls. Pediaticr Blood \& Cancer, 60(2), 309-315. doi: $10.1002 /$ pbc. 24144

Johnson-Kozlow, M., Sallis, J. F., Gilpin, E. A., Rock, C. L., \& Pierce, J. P. (2006). Comparative validation of the IPAQ and the 7-Day PAR among women diagnosed with breast cancer. The International Journal of Behavioral Nutrition and Physical Activity, 3, 7. doi: $10.1186 / 1479-5868-3-7$

Katzmarzyk, P. T., \& Lee, I. M. (2012). Sedentary behaviour and life expectancy in the USA: a cause-deleted life table analysis. BMJ OPEN, 2(4). doi: 10.1136/bmjopen-2012-000828 
Kroenke, C., Holmes, M., Feskanich, D., Chen, W., \& Colditz, G. (2005). Physical activity and survival after breast cancer diagnosis. JAMA, 293(20), 2479-2486.

Loprinzi, P. D., Cardinal, B. J., Si, Q., Bennett, J. A., \& Winters-Stone, K. M. (2012). Theorybased predictors of follow-up exercise behavior after a supervised exercise intervention in older breast cancer survivors. Supportive Care in Cancer, 20(10), 2511-2521. doi: $10.1007 / \mathrm{s} 00520-011-1360-0$

Lynch, B. M., Dunstan, D. W., Healy, G. N., Winkler, E., Eakin, E., \& Owen, N. (2010). Objectively measured physical activity and sedentary time of breast cancer survivors, and associations with adiposity: findings from NHANES (2003-2006). Cancer Causes \& Control, 21(2), 283-288. doi: 10.1007/s10552-009-9460-6

Matthews, C. E., George, S. M., Moore, S. C., Bowles, H. R., Blair, A., Park, Y., . . Schatzkin, A. (2012). Amount of time spent in sedentary behaviors and cause-specific mortality in US adults. American Journal of Clinical Nutrition, 95(2), 437-445. doi: 10.3945/ajen.111.019620

Moore, S. C., Patel, A. V., Matthews, C. E., Berrington de Gonzalez, A., Park, Y., Katki, H. A., . . Lee, I. M. (2012). Physical activity, sedentary behaviours, and the prevention of endometrial cancer. British Journal of Cancer, 103(7), 933-938. doi: 10.1038/sj.bjc.6605902

Moore, S. C., Patel, A. V., Matthews, C. E., Berrington de Gonzalez, A., Park, Y., Katki, H. A., et al. (2012). Leisure time physical activity of moderate to vigorous intensity and mortality: a large pooled cohort analysis. PLoS Medicine, 9(11), e1001335. doi: 10.1371/journal.pmed.1001335

Mutrie, N., Campbell, A. M., Whyte, F., McConnachie, A., Emslie, C., Lee, L., . . Ritchie, D. (2007). Benefits of supervised group exercise programme for women being treated for early stage breast cancer: pragmatic randomised controlled trial. British Medical Journal, 334(7592), 517. doi: 10.1136/bmj.39094.648553.AE 
National Cancer Institute. (2012). A Snapshot of Adolescent and Young Adult Cancers. Retrieved from: http://www.cancer.gov/cancertopics/aya/reports

National Cancer Institute. (2014). Cancer Topics. Cancer.gov. Retrieved from http://www.cancer.gov/cancertopics/cancerlibrary/what-is-cancer

Ottenbacher, A. J., Day, R. S., Taylor, W. C., Sharma, S. V., Sloane, R., Snyder, D. C., . . Demark-Wahnefried, W. (2011). Exercise among breast and prostate cancer survivors-what are their barriers? Journal of Cancer Survivorship, 5(4), 413-419. doi: $10.1007 / \mathrm{s} 11764-011-0184-8$

Philip, E. J., Coups, E. J., Feinstein, M. B., Park, B. J., Wilson, D. J., \& Ostroff, J. S. (2014). Physical activity preferences of early-stage lung cancer survivors. Supportive Care in Cancer, 22(2), 495-502. doi: 10.1007/s00520-013-2002-5

Pinto, B. M., Papandonatos, G. D., Goldstein, M. G., Marcus, B. H., \& Farrell, N. (2013). Homebased physical activity intervention for colorectal cancer survivors. Psychooncology, 22(1), 54-64. doi: 10.1002/pon.2047

Pinto, B. M., Rabin, C., \& Dunsiger, S. (2009). Home-based exercise among cancer survivors: adherence and its predictors. Psycho-Oncology, 18(4), 369-376. doi: 10.1002/pon.1465

Rabin, C., Horowitz, S., \& Marcus, B. (2013). Recruiting young adult cancer survivors for behavioral research. Journal of Clinical Psychology in Medical Settings, 20(1), 33-36. doi: $10.1007 / \mathrm{s} 10880-012-9317-0$

Raynor, H. A., Bond, D. S., Steeves, J., \& Thompson, D. L. (2014). Physical activity variety, energy expenditure, and body mass index. American Journal of Health Behavior, 38(4), 624-630. doi: 10.5993/AJHB.38.4.16

Reynolds, T., \& Gutman, J. (1988). Laddering theory, method, analysis, and interpretation. Journal of Advertising Research, 28(1), 11-31.

Rogers, L. Q., Hopkins-Price, P., Vicari, S., Pamenter, R., Courneya, K. S., Markwell, S., . . Lowy, M. (2009). A randomized trial to increase physical activity in breast cancer 
survivors. Medicine and Science in Sports and Exercise, 41(4), 935-946. doi:

10.1249/MSS.0b013e31818e 0e1b

Rogers, L. Q., Markwell, S., Hopkins-Price, P., Vicari, S., Courneya, K. S., Hoelzer, K., \& Verhulst, S. (2011). Reduced barriers mediated physical activity maintenance among breast cancer survivors. Journal of Sport \& Exercise Psychology, 33(2), 235-254.

Rogers, L. Q., McAuley, E., Courneya, K. S., \& Verhulst, S. J. (2008). Correlates of physical activity self-efficacy among breast cancer survivors. American Journal of Health Behavior, 32(6), 594-603. doi: 10.5555/ajhb.2008.32.6.594

Rogers, L. Q., Shah, P., Dunnington, G., Greive, A., Shanmugham, A., Dawson, B., \& Courneya, K. S. (2005). Social cognitive theory and physical activity during breast cancer treatment. Oncology Nursing Forum, 32(4), 807-815. doi: 10.1188/05.ONF.807-815

Rosenberg, R. S., Lange, W., Zebrack, B., Moulton, S., \& Kosslyn, S. M. (2014). An outdoor adventure program for young adults with cancer: positive effects on body image and psychosocial functioning. Journal of Psychosocial Oncology, 32(5), 622-636. doi: $10.1080 / 07347332.2014 .936652$

Ryan, G. J., \& Dzewaltowski, D. A. (2002). Comparing the relationships between different types of self-efficacy and physical activity in youth. Health Education \& Behavior, 29(4), 491504.

Sallis, J. F., Grossman, R. M., Pinski, R. B., Patterson, T. L., \& Nader, P. R. (1987). The development of scales to measure social support for diet and exercise behaviors. Preventive Medicine, 16(6), 825-836.

Sallis, J. F., Haskell, W. L., Wood, P. D., Fortmann, S. P., Rogers, T., Blair, S. N., \& Paffenbarger, R. S. (1985). Physical activity assessment methodology in the Five-City Project. American Journal of Epidemiology, 121(1), 91-106. 
Sallis, J. F., \& Saelens, B. E. (2000). Assessment of physical activity by self-report: status, limitations, and future directions. Research Quarterly for Exercise and Sport, 71(2 Suppl), S1-14.

Salmon, J., Owen, N., Crawford, D., Bauman, A., \& Sallis, J. F. (2003). Physical activity and sedentary behavior: a population-based study of barriers, enjoyment, and preference. Health Psychology, 22(2), 178-188.

Sarkin, J., Campbell, J., Gross, L., Roby, J., Bazzo, S., Sallis, J., and Calfas, K. (1997). Project GRAD Seven-Day Physical Activity Recall Interviewer's Manual. Medicine and Science in Sports and Exercise, 29 (Supplement), S91-S102.

Schmitz, K. H., Courneya, K. S., Matthews, C., Demark-Wahnefried, W., Galvão, D. A., Pinto, B. M., . . Medicine, A. C. o. S. (2010). American College of Sports Medicine roundtable on exercise guidelines for cancer survivors. Medicine and Science in Sports and Exercise, 42(7), 1409-1426. doi: 10.1249/MSS.0b013e3181eec112

Sellar, C. M., Bell, G. J., Haennel, R. G., Au, H. J., Chua, N., \& Courneya, K. S. (2014). Feasibility and efficacy of a 12-week supervised exercise intervention for colorectal cancer survivors. Applied Physiology Nutrition and Metabolism, 39(6), 715-723. doi: 10.1139/apnm-2013-0367

Solberg Nes, L., Liu, H., Patten, C. A., Rausch, S. M., Sloan, J. A., Garces, Y. I., . . Clark, M. M. (2012). Physical activity level and quality of life in long term lung cancer survivors. Lung Cancer, 77(3), 611-616. doi: 10.1016/j.lungcan.2012.05.096

Speed-Andrews, A. E., Stevinson, C., Belanger, L. J., Mirus, J. J., \& Courneya, K. S. (2012). Predictors of adherence to an Iyengar yoga program in breast cancer survivors. International Journal of Yoga, 5(1), 3-9. doi: 10.4103/0973-6131.91693

Sprod, L. K., Janelsins, M. C., Palesh, O. G., Carroll, J. K., Heckler, C. E., Peppone, L. J., . . Mustian, K. M. (2012). Health-related quality of life and biomarkers in breast cancer 
survivors participating in tai chi chuan. Journal of Cancer Survivorship, 6(2), 146-154. doi: $10.1007 / \mathrm{s} 11764-011-0205-7$

Stevens, B., Kagan, S., Yamada, J., Epstein, I., Beamer, M., Bilodeau, M., \& Baruchel, S. (2004). Adventure therapy for adolescents with cancer. Pediatric Blood \& Cancer, 43(3), 278284. doi: $10.1002 / \mathrm{pbc} .20060$

Sugerman, D. (2005). "I am more than my cancer": An exploratory examination of adventure programming and cancer survivors. Journal of Experiential Education, 28(1), $72-83$.

Valle, C. G., Tate, D. F., Mayer, D. K., Allicock, M., \& Cai, J. (2013). A randomized trial of a Facebook-based physical activity intervention for young adult cancer survivors. Journal of Cancer Survivorship, 7(3), 355-368. doi: 10.1007/s11764-013-0279-5

Vandelanotte, C., Sugiyama, T., Gardiner, P., \& Owen, N. (2009). Associations of leisure-time internet and computer use with overweight and obesity, physical activity and sedentary behaviors: cross-sectional study. Journal of Medical Internet Research, 11(3), e28. doi: 10.2196/jmir.1084

Wijndaele, K., Lynch, B. M., Owen, N., Dunstan, D. W., Sharp, S., \& Aitken, J. F. (2009). Television viewing time and weight gain in colorectal cancer survivors: a prospective population-based study. Cancer Causes \& Control, 20(8), 1355-1362. doi: $10.1007 / \mathrm{s} 10552-009-9356-5$

World Health Organization. Health Topics. WHO.org. Retrieved from http://www.who.int/topics/physical_activity/en/

Zebrack, B. (2009). Information and service needs for young adult cancer survivors. Supportive Care in Cancer, 17(4), 349-357. doi: 10.1007/s00520-008-0469-2

Zebrack, B., Bleyer, A., Albritton, K., Medearis, S., \& Tang, J. (2006). Assessing the health care needs of adolescent and young adult cancer patients and survivors. Cancer, 107(12), 2915-2923. doi: 10.1002/cncr.22338 


\begin{abstract}
APPENDIX A: Informed Consent
Informed Consent Form to Participate in a Research Project:

Participation Outcomes of First Descents: An Outdoor Adventure Therapy Program for Young

Adults with Cancer

A research project on participation in First Descents programs is being conducted by Dr. Marni Goldenberg in the Department of Recreation, Parks, and Tourism Administration and Liz Gill, a graduate student, in the Kinesiology Department at Cal Poly, San Luis Obispo. The purpose of the study is to understand the outcomes associated with participating in First Descents, a non-profit organization that provides free week-long outdoor adventure therapy programs to young adults with cancer.
\end{abstract}

You are being asked to take part in this study by participating in 210 -minute informal interviews and fill out a packet of questionnaires that should take approximately 15 minutes (Total time of approximately 35 minutes). The questions you will be asked concern demographic factors, your cancer and treatment, physical activity, your First Descents experience, sleep, work, and self-efficacy. You will be asked to complete the questionnaires and interview prior to your First Descents experience, as well as immediately after completion and during a follow up interview. Please be aware that you are not required to participate in this research and you may discontinue your participation at any time without penalty. You may also omit/not respond to any questions that you prefer not to answer.

There are no risks anticipated with participating in this study. Your responses will be provided confidentially to protect your privacy. A coding system will be used on research records and data will be kept secured by the researchers. Your name will not be used in any reports or this research without your permission. Potential benefits associated with the study include adding to the body of research on programs for young adult cancer survivors. This research will help clarify the outcomes and benefits of participating in First Descents, and may be 
used by organizations that develop programs for young adult cancer survivors ranging from outdoor to exercise programs.

If you have questions regarding this study or would like to be informed of the results when the study is completed, please feel free to contact Marni Goldenberg at (805) 756-7627 or mgoldenb@calpoly.edu. If you have concerns regarding the manner in which the study is conducted, you may contact Dr. Steve Davis, Chair of the Cal Poly Human Subjects Committee, at (805) 756-2754, sdavis@calpoly.edu, or Dr. Dean Wendt, Interim Dean of Research, at (805) 756-1508,dwendt@calpoly.edu.

If you agree to voluntarily participate in this research project as described, please indicate your agreement by signing below. Please keep one copy of this form for your reference, and thank you for your participation in this research.

$\begin{array}{ccc}\text { Signature of Volunteer } & & \text { Date } \\ \text { Signature of Researcher } & & \text { Date }\end{array}$

